ARTICLE OPEN

Check for updates

\title{
MAP4K4 mediates the SOX6-induced autophagy and reduces the chemosensitivity of cervical cancer
}

\author{
Hongxin Huang ${ }^{1,3}$, Qin Han ${ }^{2,3}$, Han Zheng ${ }^{1}$, Mingchen Liu' ${ }^{1}$, Shu Shi ${ }^{1}$, Ting Zhang ${ }^{1}$, Xingwen Yang ${ }^{1}$, Zhongqing Li ${ }^{1}$, Qiang Xu', \\ Hongyan Guo (iD ${ }^{2 凶}$, Fengmin Lu' and Jie Wang (iD) ${ }^{1 凶}$
}

(c) The Author(s) 2021

There are nearly $40 \%$ of cervical cancer patients showing poor response to neoadjuvant chemotherapy that can be induced by autophagy, however, the underlying mechanism has not yet been fully clarified. We previously found that Sex-determining region of $Y$-related high-mobility-group box 6 (SOX6), a tumor suppressor gene or oncogene in several cancers, could induce autophagy in cervical cancer. Accordingly, this study aims to investigate the mechanism of SOX6-induced autophagy and its potential significance in the platinum-based chemotherapy of cervical cancer. Firstly, we found that SOX6 could promote autophagy in cervical cancer cells depending on its HMG domain. Mitogen-activated protein kinase kinase kinase kinase-4 (MAP4K4) gene was identified as the direct target gene of SOX6, which was transcriptionally upregulated by binding the HMG domain of SOX6 protein to its double-binding sites within MAP4K4 gene promoter. MAP4K4 mediated the SOX6-induced autophagy through inhibiting PI3KAkt-mTOR pathway and activating MAPK/ERK pathway. Further, the sensitivity of cervical cancer cells to cisplatin chemotherapy could be reduced by the SOX6-induced autophagy in vitro and in vivo, while such a phenomenon could be turned over by autophagy-specific inhibitor and MAP4K4 inhibitor, respectively. Moreover, cisplatin itself could promote the expression of endogenous SOX6 and subsequently the MAP4K4-mediated autophagy in cervical cancer cells, which might in turn reduce the sensitivity of these cells to cisplatin treatment. These findings uncovered the underlying mechanism and potential significance of SOX6-induced autophagy, and shed new light on the usage of MAP4K4 inhibitor or autophagy-specific inhibitor for sensitizing cervical cancer cells to the platinum-based chemotherapy.

Cell Death and Disease (2022)13:13; https://doi.org/10.1038/s41419-021-04474-1

\section{INTRODUCTION}

Sex-determining region of Y-related high-mobility-group box (SOX) gene family consists of a series of homologous genes, each of which possesses a high-mobility-group (HMG) domain that migrates fast in electrophoresis [1, 2]. SOX proteins can function as transcriptional factors and regulate the transcription of multiple genes through directly binding of its HMG domain to the transcriptional regulation region of the target genes, and participate in several processes of growth and development [3-5]. According to the difference in the amino-acid sequence of HMG domain, SOX proteins can be divided into $8(\mathrm{~A}-\mathrm{H})$ subfamilies [6]. SOX6 belongs to the subfamily D of SOX protein and can recognize the conserved sequence of " $(A / T)(A / T) C A A(A /$ $\mathrm{T}) \mathrm{G}^{\prime \prime}$ within the transcriptional regulation region of the target gene with its HMG domain [5]. Apart from its role in regulating growth and development, our and other groups have found that SOX6 can also regulate the proliferation of cancer cells [7-14].

In our previous study, we find that SOX6 can inhibit the proliferation of HeLa cells, a cervical cancer cell line, through p14ARF-HDM2-p53 axis [14]. As we know, cervical cancer is related to human papillomavirus (HPV) infection, and nearly all cervical cancer patients are infected with HPV of different subtypes [15]. Although vaccination is widely used to prevent HPV infection, it will take a long period of time for the vaccine to be effectively protective, and many patients are still diagnosed at the locally advanced stage [16]. In clinical practice, the neoadjuvant chemotherapy followed by radical hysterectomy and lymphadenectomy is a viable and effective treatment option for locally advanced cervical cancer, however, there are still nearly $40 \%$ of cervical cancer patients showing poor response to neoadjuvant chemotherapy $[17,18]$. It has been reported that the sensitivity of cervical cancer cells to cisplatin chemotherapy can be reduced by autophagy [19], however, the underlying mechanisms of poor response induced by autophagy and how to increase the response of these patients have not yet been fully clarified and solved, respectively. Autophagy is a metabolic process highly conserved in eukaryotic cells, through which cells can maintain homeostasis by degrading the misfolded proteins and injured organelles [20]. Several studies have reported the dual effects of autophagy in tumorigenesis at different stages of tumor development [21, 22]. Except for its role in regulating tumorigenesis, autophagy can also

\footnotetext{
${ }^{1}$ Department of Microbiology \& Infectious Disease Center, School of Basic Medical Sciences, Peking University Health Science Center, Beijing 100191 , China. ${ }^{2}$ Department of Gynecology and Obstetrics, The Third Hospital of Peking University, Beijing 100191, China. ${ }^{3}$ These authors contributed equally: Hongxin Huang, Qin Han.

凶email: bysyghy@163.com; wangjie2015@hsc.pku.edu.cn

Edited by Professor Gian Maria Fimia
}

Received: 21 September 2021 Revised: 26 November 2021 Accepted: 10 December 2021

Published online: 20 December 2021 


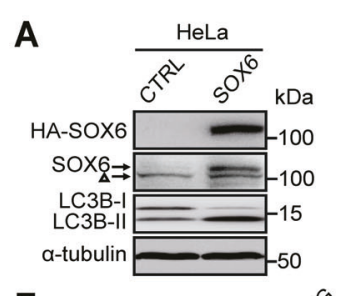

E

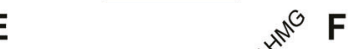

C

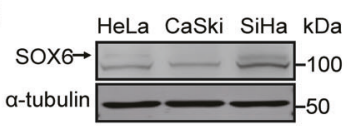

D

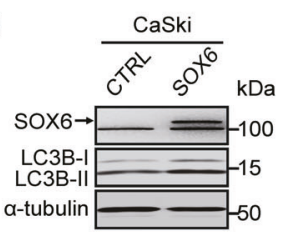

a-tubulin
$F$

G

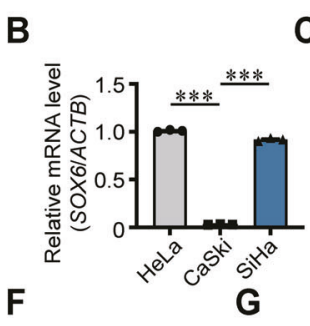

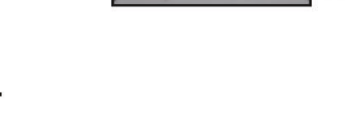

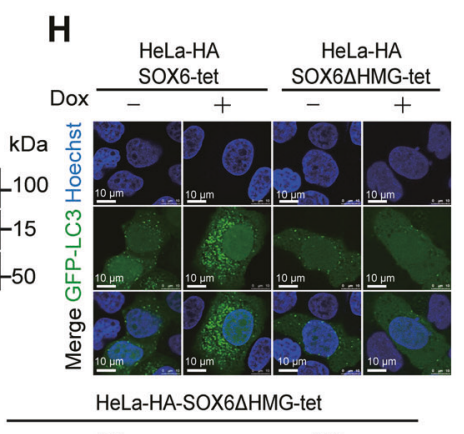

I
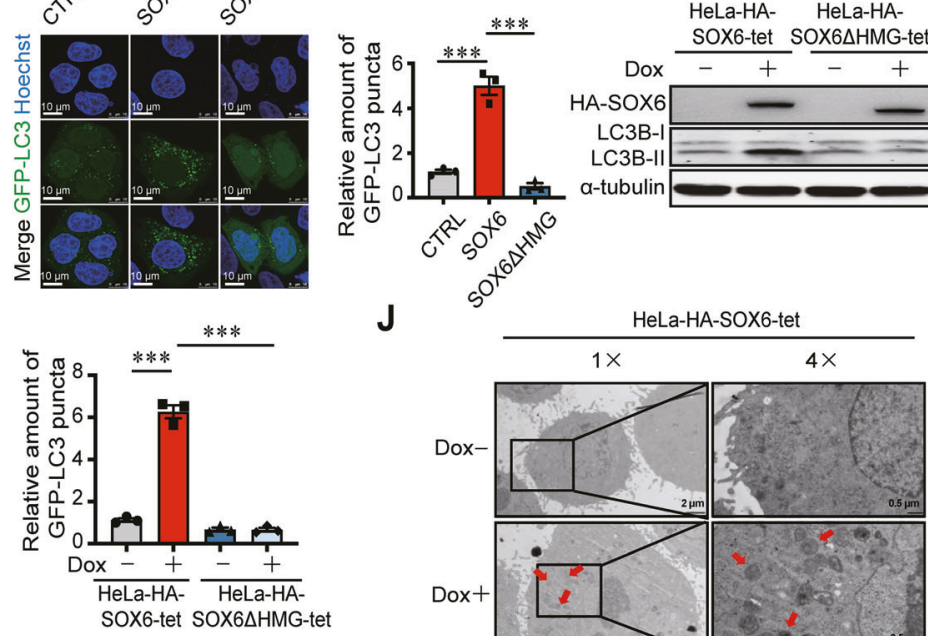

HeLa-HA-SOX6DHMG-tet
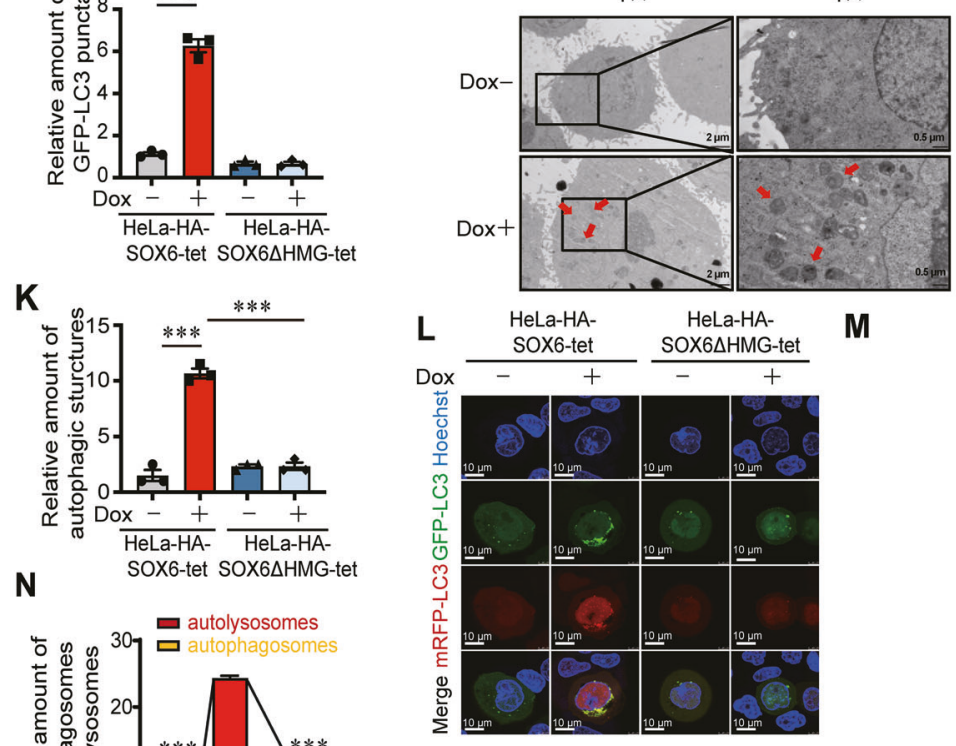

0

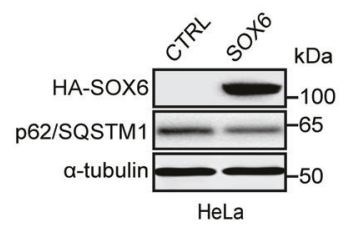

Q
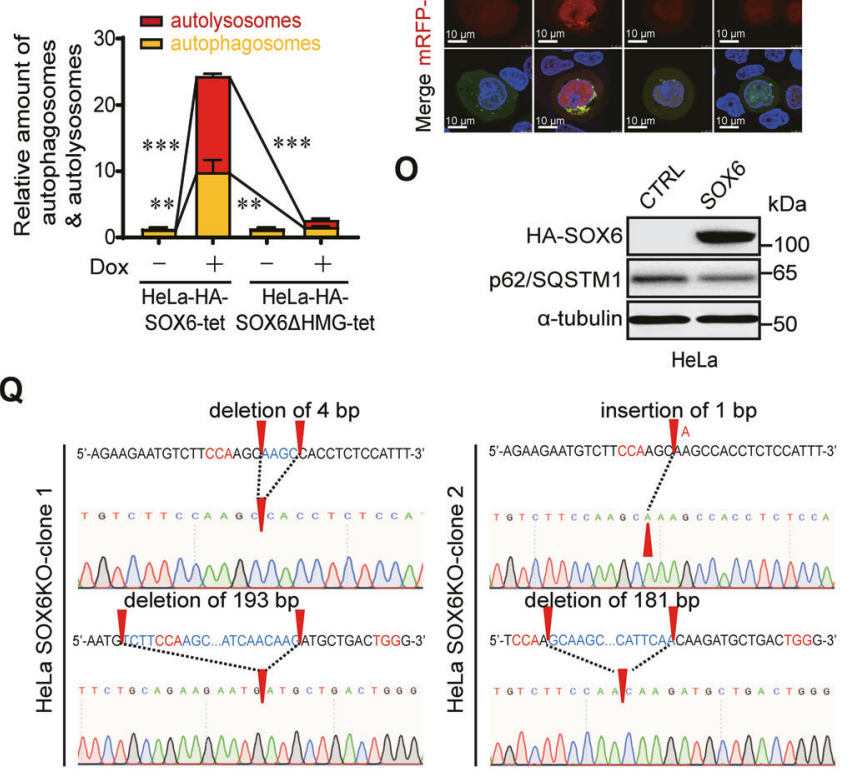

M

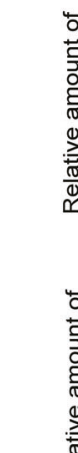

$\mathbf{R}$
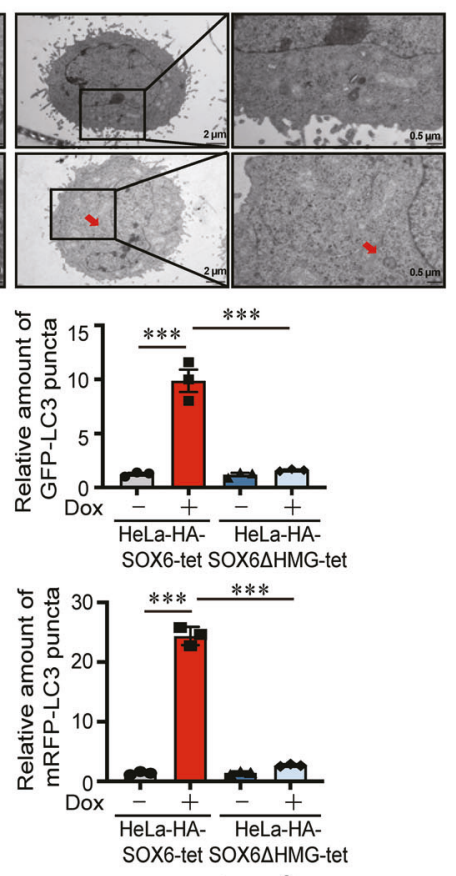

$\mathbf{P}$
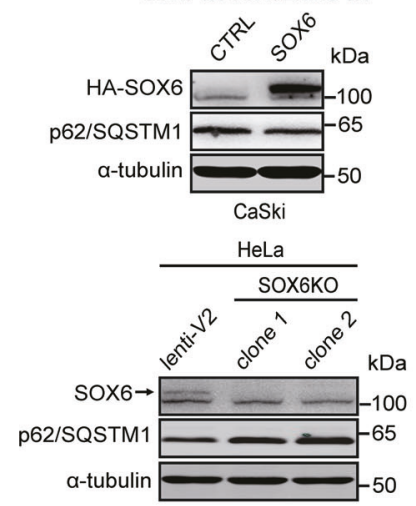

reduce the sensitivity of tumor cells to chemotherapy or even lead to chemotherapy resistance $[23,24]$.

In this study, we found that SOX6 could induce autophagy in cervical cancer cells, and mitogen-activated protein kinase kinase kinase kinase-4 (MAP4K4) gene was identified as a direct target gene of SOX6. MAP4K4, also known as hepatocyte progenitor kinase-like/germinal center kinase-like kinase (HGK) or Nckinteracting kinase (NIK), is a serine/threonine protein kinase related to S. cerevisiae Sterile 20 (STE20) [25]. Except for its role in embryonic development $[26,27]$, systemic inflammation, type 2 
Fig. 1 The effects of SOX6 in autophagosome formation and autophagic flux in cervical cancer cells. A Western blotting analysis on the level of LC3B protein in HeLa cells transfected with plex-MCS (CTRL) or plex-HA-SOX6 expression plasmid. $\alpha$-tubulin protein was used as the internal control. $\triangle$ was used to indicate the non-specific band. B The RT-qPCR (SYBR Green) analysis on the levels of SOX6 mRNA in HeLa, CaSki, and SiHa cells. $\beta$-Actin (ACTB) mRNA was used as the internal control. C Western blotting analysis on the levels of endogenous SOX6 in HeLa, CaSki, and SiHa cells. D Western blotting analysis on the level of LC3B protein in CaSki cells transfected with plex-MCS (CTRL) or plex-HASOX6 expression plasmid. E Representative confocal microscopy images and $\mathbf{F}$ relative number of GFP-LC3B puncta in HeLa cells cotransfected with GFP-LC3 and plex-MCS (CTRL), plex-HA-SOX6 or plex-HA-SOX6 $\Delta$ HMG expression plasmids. G Western blotting analysis on the level of LC3B protein in HeLa-HA-SOX6-tet and HeLa-HA-SOX6 $\Delta$ HMG-tet cells treated with or without Dox $(4 \mu \mathrm{g} / \mathrm{mL})$ for $48 \mathrm{~h}$, respectively. H Representative confocal microscopy images and I relative number of GFP-LC3B puncta in HeLa-HA-SOX6-tet and HeLa-HA-SOX6 $\Delta$ HMG-tet cells transfected with GFP-LC3 expression plasmid and treated with or without Dox $(4 \mu \mathrm{g} / \mathrm{mL})$ for $48 \mathrm{~h}$, respectively. J Transmission electron microscopy analysis and $\mathbf{K}$ relative amount of autophagic structures (indicated by red arrow) in HeLa-HA-SOX6-tet and HeLa-HA-SOX6 $\Delta$ HMGtet cells treated with or without Dox $(4 \mu \mathrm{g} / \mathrm{mL})$ for $48 \mathrm{~h}$. L Representative confocal microscopy images, M relative amount of GFP-puncta, and $\mathbf{N}$ relative amount of autophagosomes (GFP-positive, RFP-positive) and autolysosomes (GFP-negative, RFP-positive) in HeLa-HA-SOX6-tet and HeLa-HA-SOX6 $\Delta$ HMG-tet cells transfected with mRFP-GFP-LC3 adenovirus reporter and treated with or without Dox (4 $\mu \mathrm{g} / \mathrm{mL})$ treatment for $48 \mathrm{~h}$, respectively. O Western blotting analysis on the level of p62/SQSTM1 protein in HeLa cells and P in CaSki cells transfected with plex-MCS (CTRL) or plex-HA-SOX6 expression plasmid. Q The direct sequencing results on the PCR product of SOX6 gene from two HeLa-SOX6KO clones. The red arrow head indicates the location of the indel (insertion-deletion) mutation in each allele. $\mathbf{R}$ Western blotting analysis on the levels of p62/SQSTM1 in two HeLa-SOX6KO clones compared with the control HeLa cell (lenti-V2). Data are mean \pm SEM of three independent experiments or at least 50 cells scored $\left({ }^{* *} P<0.01,{ }^{* * *} P<0.001\right.$, one-way ANOVA and post hoc Tukey tests).

diabetes and atherosclerosis, MAP4K4 also participates in tumor development and is a negative prognostic indicator in several cancers, including hepatocellular carcinoma, lung adenocarcinoma and prostate cancer [28-34]. However, little is known about its role in autophagy and its value in cancer therapy. In this study, we further found that MAP4K4 could mediate the SOX6-induced autophagy through inhibiting PI3K-Akt-mTOR pathway and activating MAPK/ERK pathway, which could reduce the sensitivity of cervical cancer cells to cisplatin chemotherapy in vitro and in vivo.

\section{RESULTS \\ SOX6 promotes autophagosome formation and autophagic flux depending on its HMG domain}

It has been reported that SOX6 can inhibit the proliferation of HeLa cells in our previous study [14]. In this study, we further found that the level of LC3B-II protein, an autophagy-specific protein, could be increased by overexpressing SOX6 in HeLa cells (Fig. 1A). Since LC3B-II was located on the membrane of autophagosomes and lipidated from the cytosolic LC3B-I, the increased ratio of LC3B-II to LC3B-I (LC3B-II/LC3B-I) induced by SOX6 indicated that SOX6 could promote the formation of autophagosomes (Fig. 1A). Based on the fact that the level of endogenous SOX6 in CaSki cells was far lower than that in HeLa and SiHa cells (Fig. 1B, C), CaSki cells were also chosen to further explore the effect of SOX6 in autophagy of cervical cancer cells. Consistently, SOX6 could also increase the level of LC3B-II/LC3B-I in CaSki cells (Fig. 1D). Further, confocal microscopy analysis showed that the number of GFP-LC3B puncta in HeLa cells overexpressing SOX6 was far more than that in the vector control cells, whereas such a phenomenon was disappeared when its HMG domain was deleted (Fig. 1E, F). Consistently, doxycycline (Dox)-induced SOX6 protein could also increase the level of LC3BII/LC3B-I and the number of GFP-LC3B puncta in HeLa-HA-SOX6tet cells, but not for the SOX6 protein with HMG domain deleted (SOX6 $\triangle \mathrm{HMG}$ ) in HeLa-HA-SOX6 $\mathrm{HMG}$-tet cells (Fig. 1G-I). Moreover, transmission electron microscopy analysis revealed that the Dox-induced SOX6 could increase the number of autophagosomeor autolysosome-like structures in HeLa-HA-SOX6-tet cells, but not in HeLa-HA-SOX6 $\triangle$ HMG-tet cells (Fig. 1J, K).

Autophagic flux is a dynamic process, including autophagosome formation, autolysosome formation and substrate degradation. To investigate the role of SOX6 in the whole process of autophagic flux, the mRFP-GFP-LC3 adenovirus reporter was used in this study. The result revealed that the number of both yellow (autophagosome) and red puncta (autolysosome) were increased in Dox-treated HeLa-HA-SOX6-tet cells, but not in HeLa-HASOX6 6 HMG-tet cells, suggesting that SOX6 could promote the formation of both autophagosome and autolysosome (Fig. $1 \mathrm{~L}-\mathrm{N}$ ). As a substrate, p62/SQSTM1 protein is preferentially degraded in autolysosomes [35]. Therefore, to further investigate the role of SOX6 in substrate degradation via autolysosomes, the impact of SOX6 on the level of p62/SQSTM1 protein was analyzed. The result revealed that SOX6 could also significantly reduce the level of p62/SQSTM1 (Fig. 10, P). Further, SOX6 gene was knocked out by CRISPR/Cas9 genome editing approach in HeLa cells, and two cell clones with heterozygous insertion-or-deletion (indel) mutations were successfully established (Fig. 1Q). Accompanied by the loss of endogenous SOX6 expression, the autophagic degradation of p62/SQSTM1 was blocked in both HeLa-SOX6KO cell clones (Fig. 1R).

\section{MAP4K4 gene is the potential target gene mediating the SOX6-induced autophagy}

To investigate the underlying mechanism responsible for SOX6induced autophagy, genome-wide expression profile of HeLa cells with or without SOX6 overexpression were analyzed. A total of 4488 differentially expressed genes (DEGs) were identified by microarray, and were subsequently analyzed by KEGG pathway enrichment analysis using the Database for Annotation, Visualization and Integrated Discovery (DAVID). The top 20 enriched pathways were listed, and 6 of them were related to autophagy, including PI3K-Akt, MAPK, lysosomal, p53, FoxO, and phagosome pathways (Fig. 2A). According to the number of DEGs, there were most DEGs enriched in PI3K-Akt pathway, followed by MAPK pathway among pathways associated with autophagic machinery (Supplementary Fig. S1). Next, we confirmed that SOX6 could inhibit PI3K-Akt-mTOR pathway, but activate MAPK/ERK pathway in HeLa and CaSki cells (Fig. 2B and Supplementary Fig. S2).

To screen the direct target gene of SOX6 involved in regulating the above two pathways, a total of 2836 DEGs between the HeLaHA-SOX6-tet cells with and without Dox treatment were further identified by RNA-sequencing analysis (Fig. 2C). Further, 32 overlapping DEGs were screened out by merging the DEGs enriched in PI3K-Akt and MAPK pathways between microarray and RNA-sequencing analysis (Fig. 2D), which were listed in heatmap (Fig. 2E). Among them, MAP4K4 gene is at the upstream of MAPK signaling pathway and has been reported to regulate both $\mathrm{PI} 3 \mathrm{~K}$ Akt and MAPK pathways $[36,37]$, indicating that MAP4K4 gene might be the target gene of SOX6 in regulating both pathways. Next, we confirmed that SOX6 could upregulate both mRNA and protein levels of MAP4K4 depending on its HMG domain, which confirmed the results of microarray and RNA-sequencing analyses 

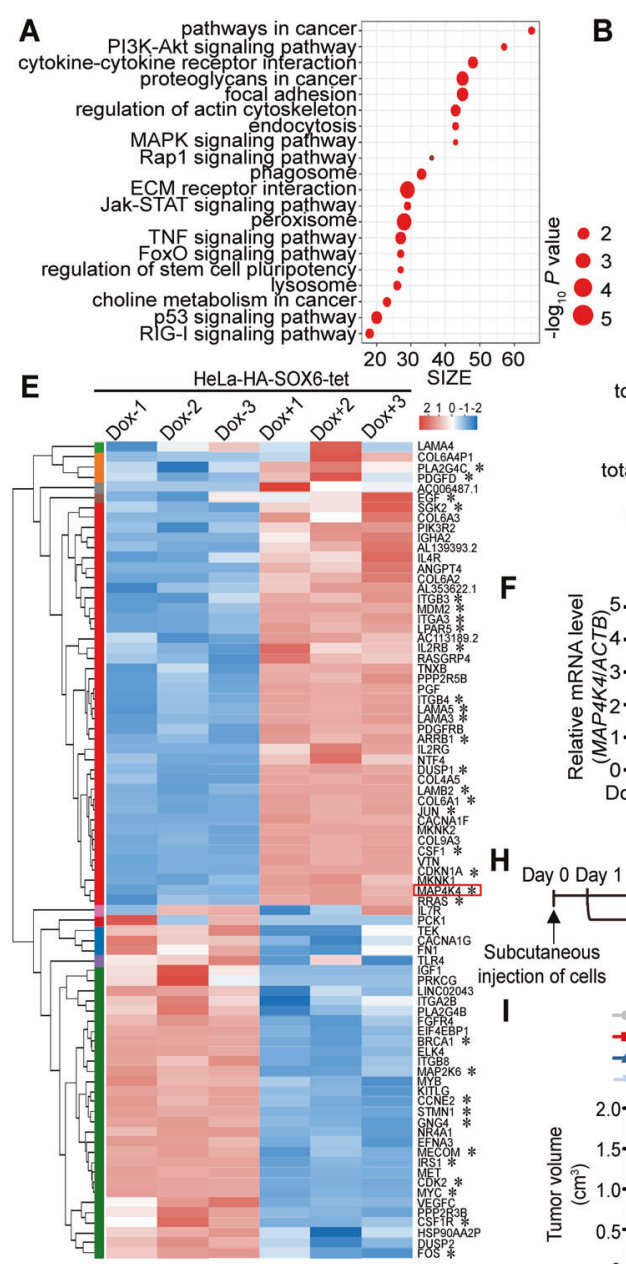

B

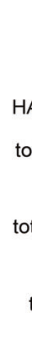

total ER-SO
p-ER
total
p-PI3

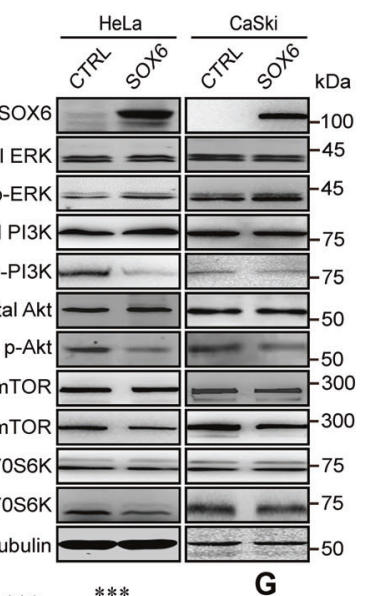

$F$
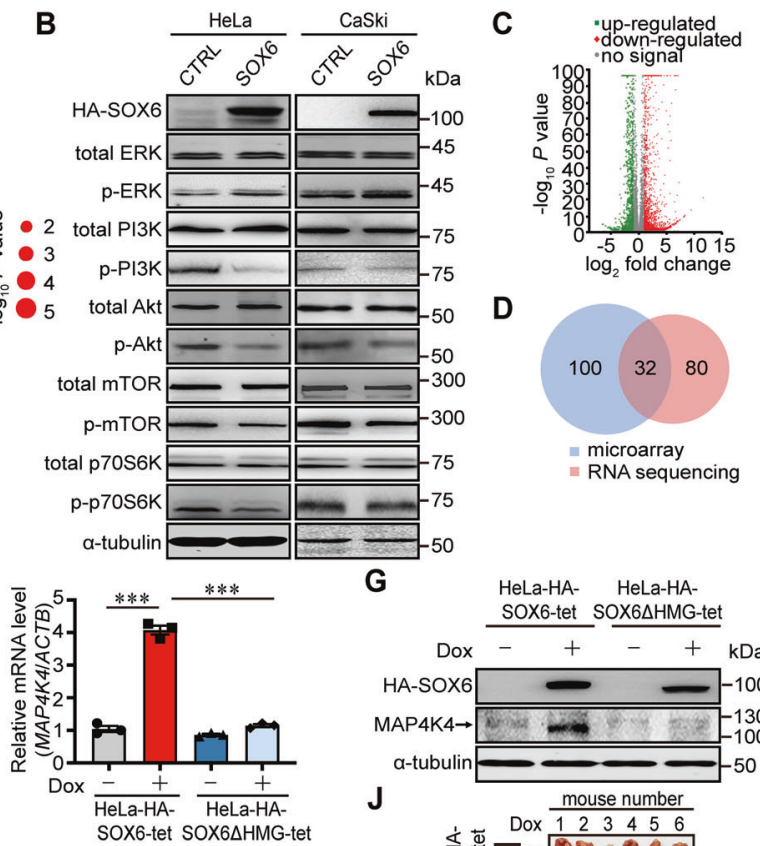

D

$\log _{2}$ fold change

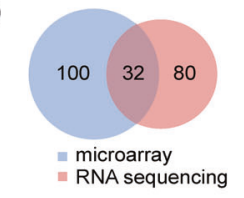

HeLa-HA- HeLa-HA-

SOX6-tet SOX6AHMG-tet

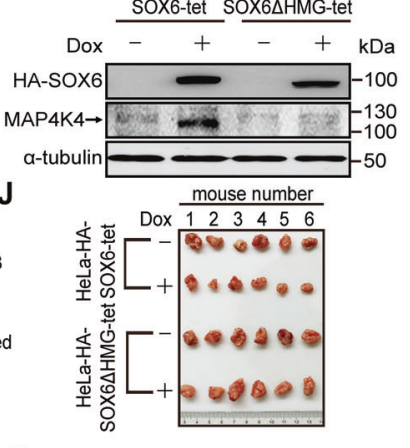

K
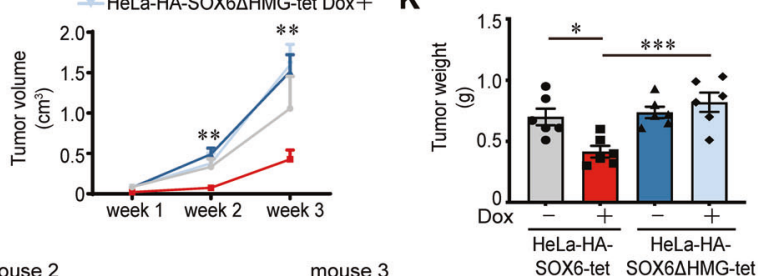

$\mathbf{L}$
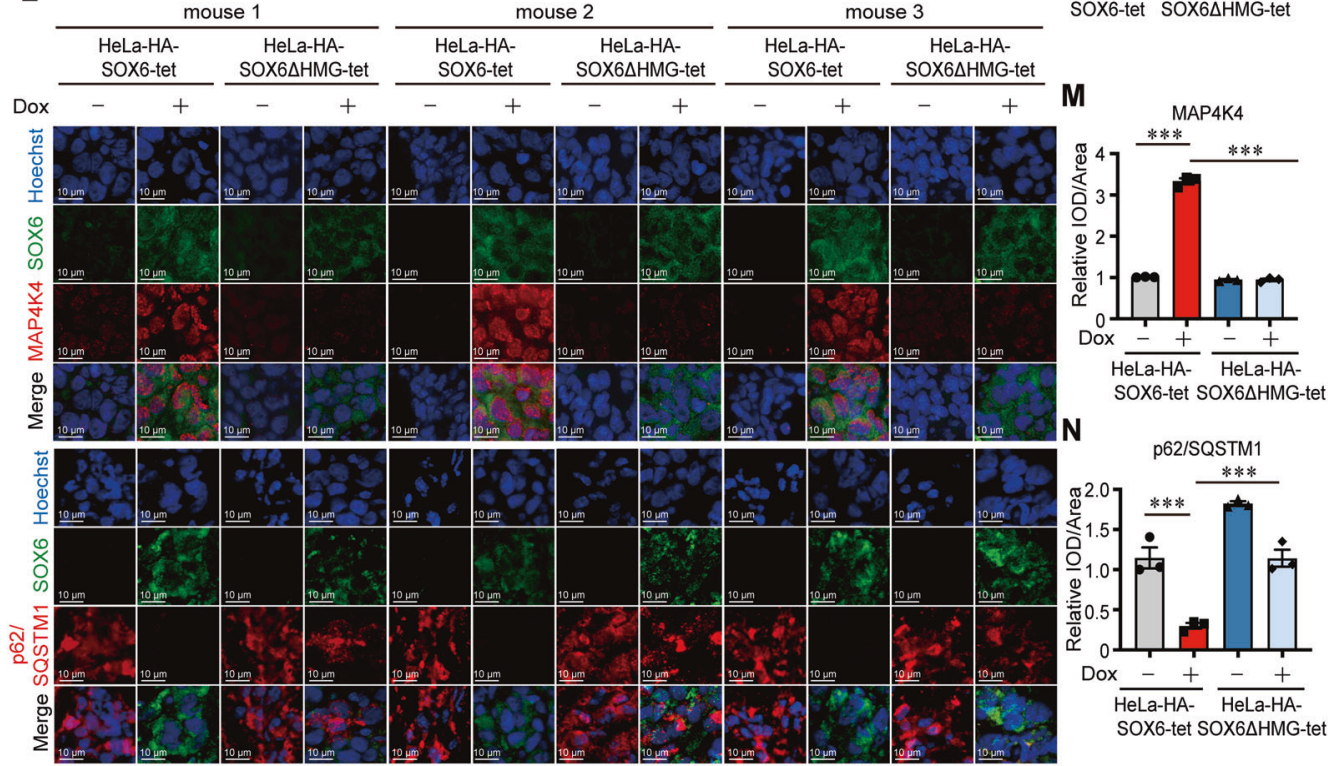

(Fig. 2F, G, Supplementary Fig. S3A, B). Moreover, the dualluciferase assay showed that SOX6 could increase the transcriptional activity of the MAP4K4 gene promoter depending on its HMG domain, suggesting that SOX6 promoted the expression of MAP4K4 gene at transcriptional level (Supplementary Fig. S4A-C).
Next, the effects of SOX6 in autophagy and the expression of MAP4K4 were further investigated in vivo (Fig. $2 \mathrm{H}$ ). The result revealed that the Dox-induced SOX 6 could significantly reduce the size of tumors in the mice injected with HeLa-HA-SOX6-tet cells at both 2- and 3-weeks post-injection, but not for SOX6 $\Delta \mathrm{HMG}$ in the 
Fig. 2 The potential target gene mediating the SOX6-induced autophagy. A Scatter plot of top 20 enriched KEGG pathways regulated by SOX6, according to the differentially expressed genes (DEGs) of microarray data. B Western blotting analysis on the protein levels of key molecules within MAPK/ERK and PI3K-Akt signaling pathways in HeLa and CaSki cells transfected with plex-MCS (CTRL) or plex-HA-SOX6 expression plasmid. $\alpha$-tubulin protein was used as the internal control. C Overview of the SOX6-regulated DEGs by RNA sequencing. D Venn diagram of the DEGs enriched in MAPK and PI3K-Akt signaling pathways between microarray and RNA-sequencing. E Heatmap of the 32 DEGs merged between microarray and RNA-sequencing analysis. The color scale beside the heatmap represents the raw $Z$-score ranging from blue (low expression) to red (high expression). F The RT-qPCR (SYBR Green) analyses on the levels of MAP4K4 mRNA in HeLa-HA-SOX6-tet and HeLa-HA-SOX6 $\Delta$ HMG-tet cells treated with or without Dox $(4 \mu \mathrm{g} / \mathrm{mL})$ for $48 \mathrm{~h}$, respectively. G Western blotting analyses on the levels of MAP4K4 protein in HeLa-HA-SOX6-tet and HeLa-HA-SOX6 $\Delta$ HMG-tet cells treated with or without Dox $(4 \mu \mathrm{g} / \mathrm{mL})$ for $48 \mathrm{~h}$, respectively. $\mathbf{H}$ Experimental design of the steps used to produce xenograft in BALB/c nude mice. I Growth curve of tumors formed by subcutaneous injection of HeLa-HA-SOX6-tet or HeLa-HA-SOX6 $\Delta$ HMG-tet cells into the left flank of nude mice, after which the mice were daily intraperitoneally injected with Dox ( $20 \mathrm{mg} / \mathrm{kg}$, PBS as solvent control) for 3 weeks. J Representative tumor blocks that were collected from the mice sacrificed under anesthesia at 3 weeks post-injection. K Average tumor weights $(\mathrm{g})$ of tumor blocks. $\mathbf{L}$ Immunofluorescence staining of SOX6 (green) and MAP4K4 (red), or SOX6 (green) and p62/SQSTM1 (red) in the frozen section of xenograft tumor tissues. Data are mean \pm SEM of at least three fields. M Relative expression level of SOX6 (green) and MAP4K4 (red) or N SOX6 (green) and p62/SQSTM1 (red) in the frozen section of xenograft tumor tissues. All data are mean $\pm \mathrm{SEM}\left({ }^{*} P<0.05,{ }^{* * *} P<0.001\right.$, one-way ANOVA and post hoc Tukey tests). IOD integral optical density.

mice injected with HeLa-HA-SOX6 $\Delta \mathrm{HMG}$-tet cells (Fig. 2I). Consistently, in the mice injected with HeLa-HA-SOX6-tet cells, the sizes and weights of tumor blocks collected at 3 weeks postinjection were significantly lower in the Dox-treated group than that in PBS-treated group, but not for the mice injected with HeLaHA-SOX6 $\triangle$ HMG-tet cells (Fig. 2J, K). Further, SOX6 could also significantly downregulate the level of p62/SQSTM1 protein and upregulate the level of MAP4K4 protein depending on its HMG domain (Fig. 2L-N), which was further confirmed by western blotting analysis (Supplementary Fig. S5).

\section{MAP4K4 gene is the direct target gene of SOX6 and mediates the SOX6-induced autophagy}

Since SOX6-induced autophagy depended on its HMG domain, we further investigated whether MAP4K4 gene was the direct target gene of SOX6. Firstly, the consensus sequence recognized by HMG domain of SOX6 protein was searched in the promoter region of MAP4K4 gene. The results revealed that two potential binding sites were identified at $59-77$ and $430-444 \mathrm{bp}$ upstream of the transcriptional start site (TSS) of MAP4K4 gene, respectively (Fig. 3A). Next, chromatin immunoprecipitation (ChIP) combined with PCR assay confirmed that the sequences containing doublebinding sites could be bound by the HMG domain of SOX6 protein (Fig. 3B). Further, the dual-luciferase assay showed that SOX6 could significantly enhance the transcriptional activity of MAP4K4 gene promoter depending on its HMG domain, whereas such an effect was reduced when one binding site was mutated, and was even disappeared when both binding sites were mutated (Fig. 3C). Taken together, the transcriptional activity of MAP4K4 gene promoter could be enhanced by binding of SOX 6 protein to the two binding sites in the promoter of MAP4K4 gene.

Moreover, the level of LC3B-II/LC3B-I was remarkably reduced when the expression of endogenous MAP4K4 was knocked down by MAP4K4-specific small interfering RNAs (siMAP4K4) (Fig. 3D and Supplementary Fig. S6A) or inhibited by MAP4K4-specific inhibitor (PF-06260933) (Fig. 3E). Similarly, the SOX6-induced autophagosome formation was also inhibited by transfecting siMAP4K4 (Supplementary Fig. S6B) or treating with PF-06260933 (Fig. 3F, G). Above results suggested that MAP4K4 could mediate the SOX6-induced autophagy.

\section{MAP4K4 mediates the SOX6-induced activation of MAPK/ERK} pathway and inhibition of PI3K-Akt-mTOR pathway

Since SOX6 could regulate PI3K-Akt-mTOR and MAPK/ERK pathways, and both pathways have been reported to be associated with autophagy [38, 39], we further investigated whether these two pathways were involved in the SOX6-induced autophagy and were regulated by MAP4K4. The result revealed that the Doxinduced SOX6 could activate MAPK/ERK pathway and inhibit PI3K-
Akt-mTOR pathway in HeLa-HA-SOX6-tet cells, but not for SOX6 $\mathrm{HMG}$ in HeLa-HA-SOX6 $\triangle \mathrm{HMG}$-tet cells (Fig. $4 \mathrm{~A}$ and Supplementary Fig. S7A-E). Further, the SOX6-induced activation of MAPK/ERK pathway and inhibition of PI3K-Akt-mTOR pathway, as well as autophagy were turned over when the expression of endogenous MAP4K4 was knocked down by siMAP4K4 (Fig. 4B and Supplementary Fig. S7F-J) or inhibited by PF-06260933 (Fig. $4 C)$.

Meanwhile, the SOX6-mediated activation of MAPK/ERK pathway and inhibition of PI3K-Akt-mTOR pathway could be enhanced by ectopic MAP4K4 (Fig. 4D). Moreover, MAP4K4 could also directly activate MAPK/ERK pathway and inhibit PI3K-Akt-mTOR pathway (Fig. 4E), as well as promote the formation of autophagosomes in HeLa cells (Fig. 4F). It suggested that MAP4K4 could mediate the SOX6-induced autophagy through activating MAPK/ERK pathway and inhibiting PI3K-Akt-mTOR pathway.

\section{SOX6-induced autophagy reduces the sensitivity of cervical cancer cells to cisplatin treatment}

It has been reported that the cisplatin-mediated anticancer effects are associated with several intertwined mechanisms including DNA damage and subsequent apoptosis, but autophagy could lead to the occurrence of chemoresistance through decreasing the cisplatin-induced apoptosis [40-42]. In this study, we found that some of top 20 Gene Ontology (GO)-enriched terms of MAP4K4 gene were related to the regulations of apoptosis and cell death (Fig. 5A). Therefore, to further investigate whether the SOX6induced autophagy affected the therapeutic effect of cisplatin in cervical cancer cells, the effect of SOX6 in the cisplatin-induced apoptosis was firstly analyzed by flow cytometry analysis. The result revealed that SOX6, other than SOX6 $\Delta \mathrm{HMG}$, could significantly reduce the percentage of cisplatin-induced apoptotic cells (Fig. 5B, C). Further, western blotting analysis confirmed that SOX6 could significantly reduce the levels of apoptosis-related proteins in HeLa-HA-SOX6-tet cells (Fig. 5D), but not for SOX6 $\triangle \mathrm{HMG}$ in HeLa-HA-SOX6 $\Delta \mathrm{HMG}$-tet cells (Supplementary Fig. S8A).

Next, we further investigated the impact of SOX6-induced autophagy on the chemosensitivity of cisplatin treatment in xenograft (Fig. 5E). The results revealed that the sizes of tumors were significantly reduced at both 2- and 3-weeks post-injection under cisplatin treatment in PBS group, whereas the reduced tumor sizes under cisplatin treatment were significantly lower in Dox-treated group than that in PBS-treated group, indicating that SOX6 could significantly reduce the chemosensitivity of xenograft to cisplatin (Fig. 5F and Supplementary S8B-G). To further investigate the role of SOX6-induced autophagy, the frozen sections of xenograft samples were used for immunofluorescence staining. The results revealed that SOX6 could also induce 
A

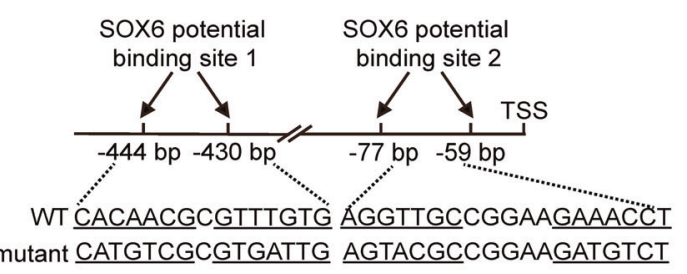

B

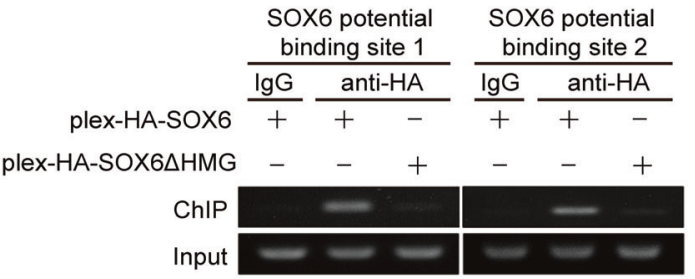

D

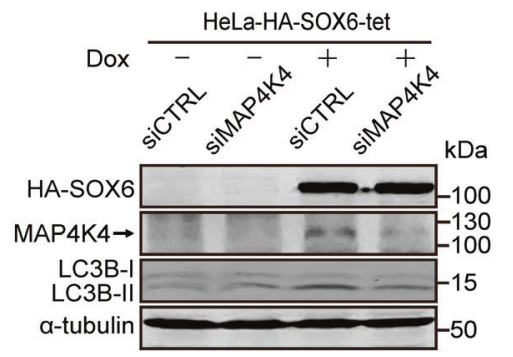

G

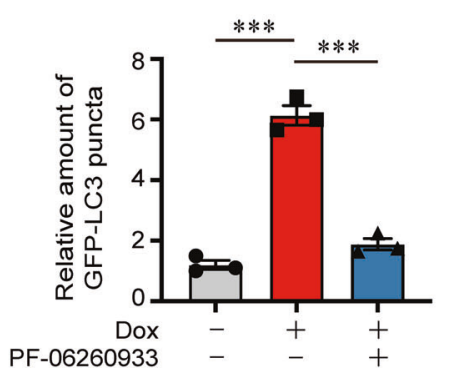

Fig. 3 The role of MAP4K4 in the SOX6-induced autophagy. A Schematic representation on two potential SOX6 double-binding sites in the promoter region of MAP4K4 gene. B The binding activity of SOX6 to the two potential double-binding sites in the promoter of MAP4K4 gene was analyzed by ChIP-PCR. C Dual-luciferase assay analysis on the effects of SOX6 in the transcriptional activity of wild-type (WT) and mutant (mut 1 and mut $1+2$ ) MAP4K4 gene promoter in HeLa cells. D Western blotting analysis on the levels of MAP4K4 and LC3B proteins regulated by SOX6 in HeLa-HA-SOX6-tet cells that the expression of endogenous MAP4K4 was knocked down by transfecting siMAP4K4 or E treating with small molecule inhibitor, PF-06260933 $(3 \mu \mathrm{M})$. $\alpha$-tubulin protein was used as the internal control. F Representative confocal microscopy images and G relative number of GFP-LC3 puncta in HeLa-HA-SOX6-tet cells transfected with GFP-LC3 expression plasmid and treated with or without Dox $(4 \mu \mathrm{g} / \mathrm{mL})$ and/or PF-06260933 $(3 \mu \mathrm{M})$ for $48 \mathrm{~h}$. Data are mean \pm SEM and at least 50 cells scored. ${ }^{* * * P}<0.001, N S$ non-significant, one-way ANOVA and post hoc Tukey tests).

autophagy in vivo, which was indicated by the decreased level of p62/SQSTM1 (Fig. 5G, H). Meanwhile, the SOX6-induced autophagy could significantly reduce the percentage of apoptotic (TUNEL-positive) cells induced by cisplatin treatment (Fig. 5l, J). These results suggested that the SOX6-induced autophagy could also reduce the chemosensitivity of xenograft to cisplatin in vivo.

\section{The sensitivity of cervical cancer cells to cisplatin treatment can be increased by inhibiting autophagy and MAP4K4 expression}

To further investigate the role of SOX6-induced autophagy in the response to cisplatin treatment, we explored the impact of cisplatin treatment on the expression of endogenous SOX6. The results revealed that the level of endogenous SOX6 was significantly increased under cisplatin treatment, and accompanied with the increased level of MAP4K4, activation of MAPK/ERK, inhibition PI3K-Akt-mTOR pathway and induction of autophagy in both HeLa and SiHa cells (Fig. 6A, B and Supplementary Fig. S9). Accompanied with the lower levels of endogenous SOX6 protein and the higher level of p62/SQSTM1 protein in CaSki cells compared to HeLa cells under cisplatin treatment, the sensitivity to cisplatin was higher in CaSki cells than that in HeLa cells, indicating the possibility that the cisplatin-induced higher level of endogenous SOX6 might contribute to the lower sensitivity to cisplatin in HeLa cells (Fig. 6C). This possibility was further confirmed by the results that the sensitivity to cisplatin was significantly increased when the endogenous SOX6 was knocked out in HeLa cells (Fig. 6D-F).

Next, to further verify the clinical significance of SOX6 and MAP4K4 protein levels in cervical cancer patients with routine cisplatin treatment, we collected the cervical cancer tissues from 14 cervical cancer patients after receiving routine cisplatin treatment, half of whom were sensitive to chemotherapy according to the new guidelines to evaluate the response to treatment in solid tumors [43]. The corresponding clinical characteristics were shown in Supplementary Fig. S10. The results revealed that the levels of SOX6 protein and its downstream MAP4K4 protein in the frozen cervical cancer tissues of patients who were sensitive to cisplatin were lower than those in patients who were not sensitive to cisplatin (Fig. 6G-I). Meanwhile, the level of p62/SQSTM1 protein in cervical cancer patients who were sensitive to cisplatin was higher than that in patients who were not sensitive to cisplatin, which further confirmed that the higher level of SOX6-induced autophagy mediated by MAP4K4 might reduce the sensitivity of cervical cancer cells to cisplatin chemotherapy in cervical cancer patients (Fig. 6G, J, K). 
A

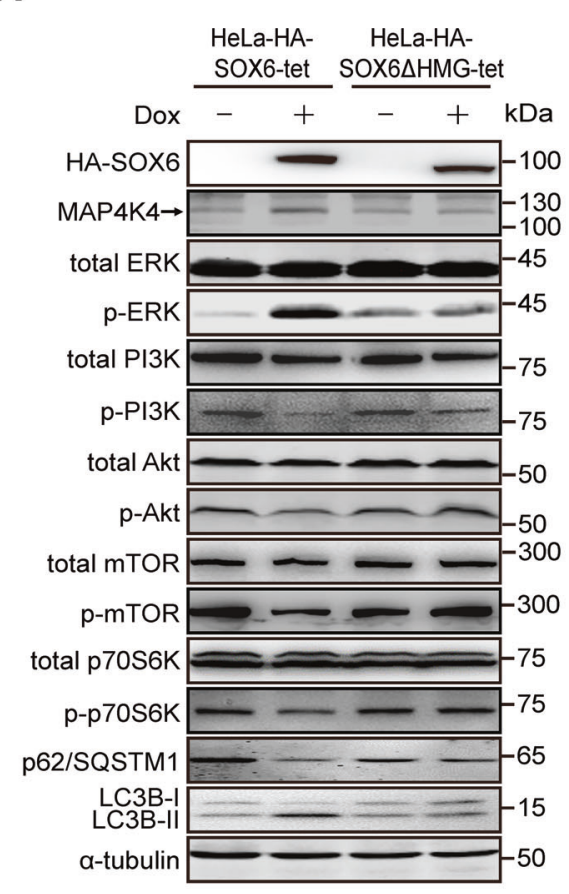

D

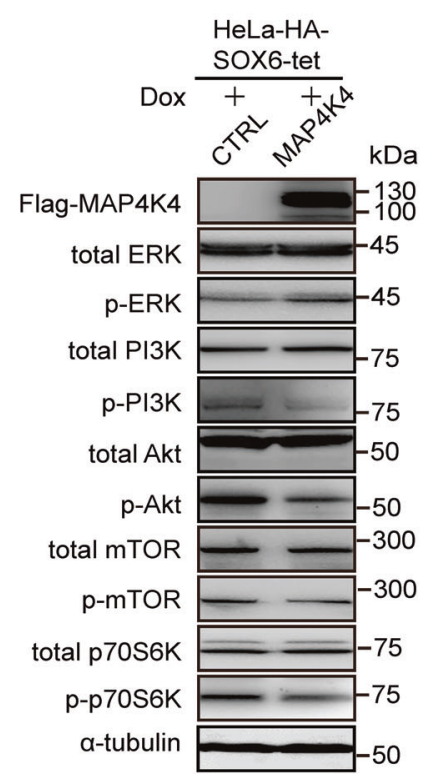

B

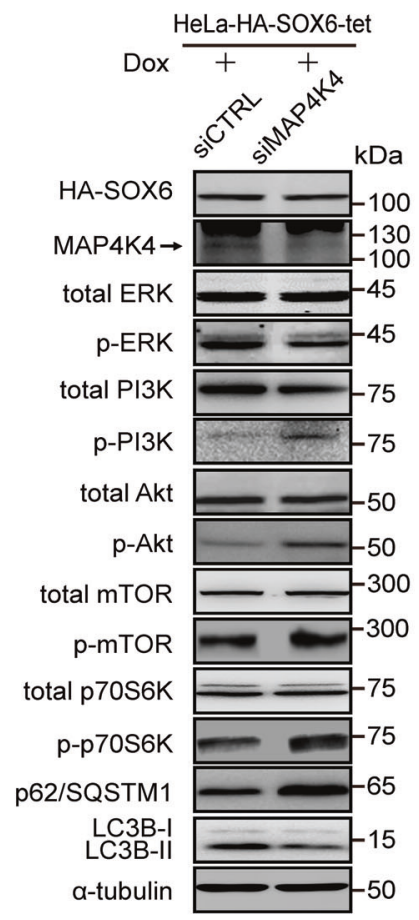

$\mathbf{E}$

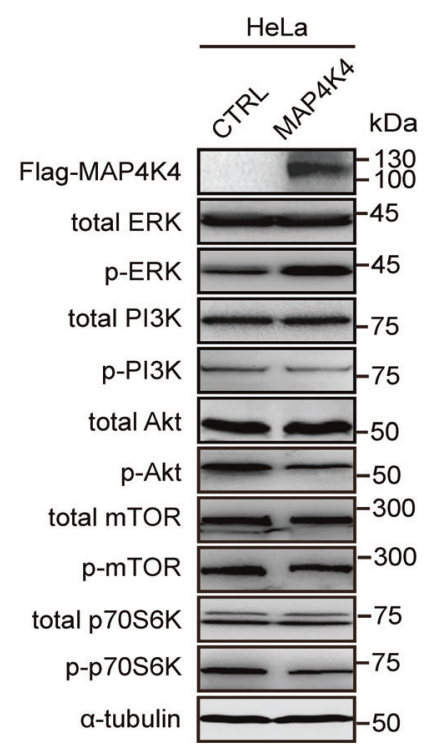

C

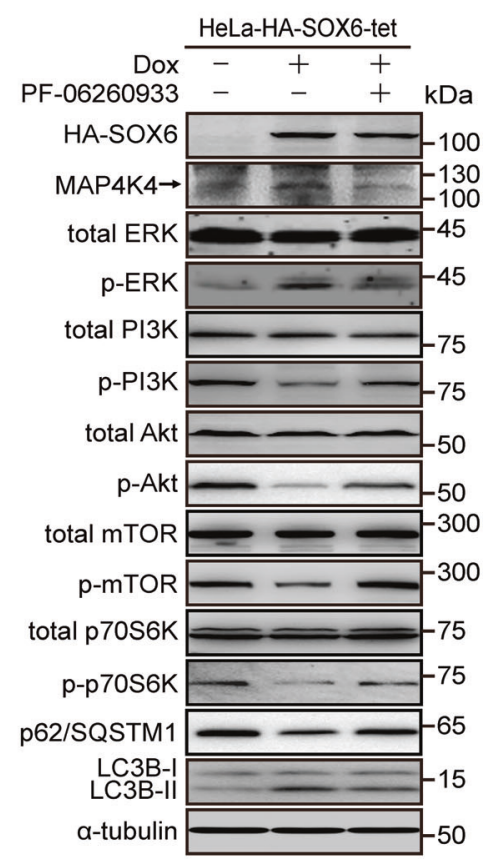

$\mathbf{F}$
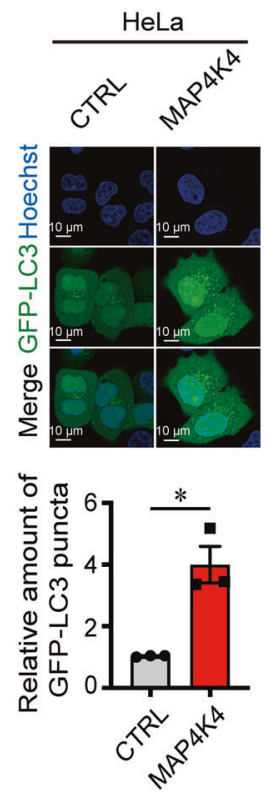

Fig. 4 The role of MAP4K4 in the SOX6-induced activation of MAPK/ERK pathway and inhibition of PI3K-Akt-mTOR pathway. A Western blotting analysis on the protein levels of key molecules within MAPK/ERK and PI3K-Akt-mTOR signaling pathways in HeLa-HA-SOX6-tet and HeLa-HA-SOX6 $\triangle$ HMG-tet cells. B Western blotting analysis on the protein levels of key molecules within MAPK/ERK and PI3K-Akt-mTOR signaling pathways in HeLa-HA-SOX6-tet cells that the endogenous MAP4K4 was knocked down by transfecting siMAP4K4 or C treating with small molecule inhibitor, PF-06260933 (3 $\mathrm{MM}$ ). D Western blotting analysis on the protein levels of key molecules within MAPK/ERK and PI3KAkt-mTOR signaling pathways in HeLa-HA-SOX6-tet cells transfected with pCDH-MCS (CTRL) or pCDH-Flag-MAP4K4 expression plasmids under Dox $(4 \mu \mathrm{g} / \mathrm{mL})$ treatment for $48 \mathrm{~h}$. E Western blotting analysis on the protein levels of key molecules within MAPK/ERK and PI3K-AktmTOR signaling pathways in HeLa cells transfected with pCDH-MCS (CTRL) or PCDH-Flag-MAP4K4 expression plasmids, respectively. $\alpha$-tubulin protein was used as the internal control. F Representative confocal microscopy images of HeLa cells co-transfected with GFP-LC3 and pCDHMCS (CTRL) or PCDH-Flag-MAP4K4 expression plasmids. Data are mean \pm SEM and at least 50 cells scored. ( ${ }^{*} P<0.05$, Student's $t$-test, two tails).

Based on the phenomenon that the SOX6-induced autophagy mediated by MAP4K4 could reduce the sensitivity of cervical cancer cells to cisplatin treatment, we further investigated whether the sensitivity to cisplatin could be increased by inhibiting autophagy or MAP4K4 expression. Firstly, Baf A1 was used to inhibit the SOX6-induced autophagy. The result revealed that the SOX6-reduced sensitivity of HeLa-HA-SOX6-tet cells to cisplatin was turned over under Baf A1 treatment (Fig. 7A).

Further, the sensitivity of HeLa cells to cisplatin could also be increased by inhibiting the expression of endogenous MAP4K4 
A

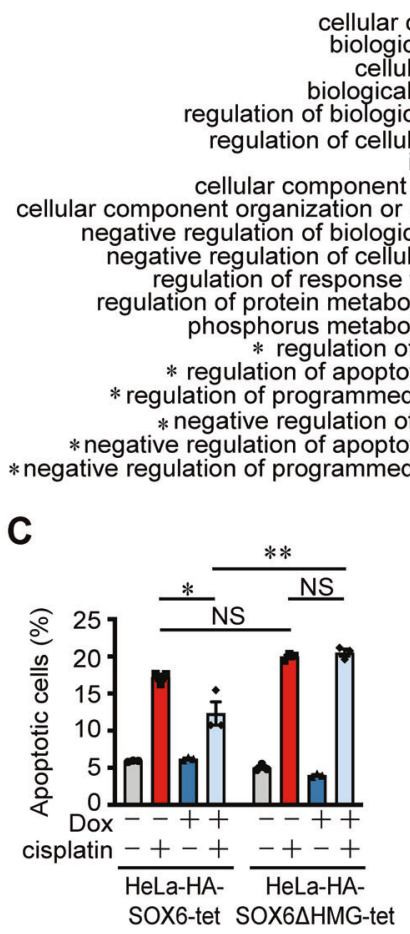

E

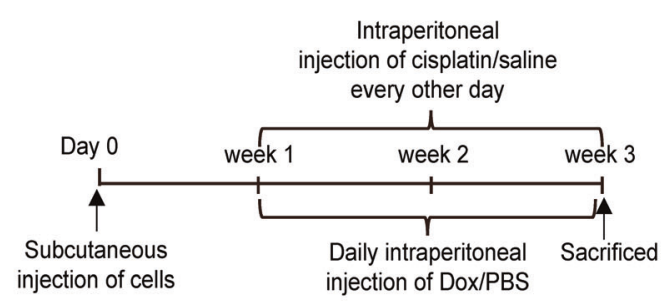

H p62/SQSTM1
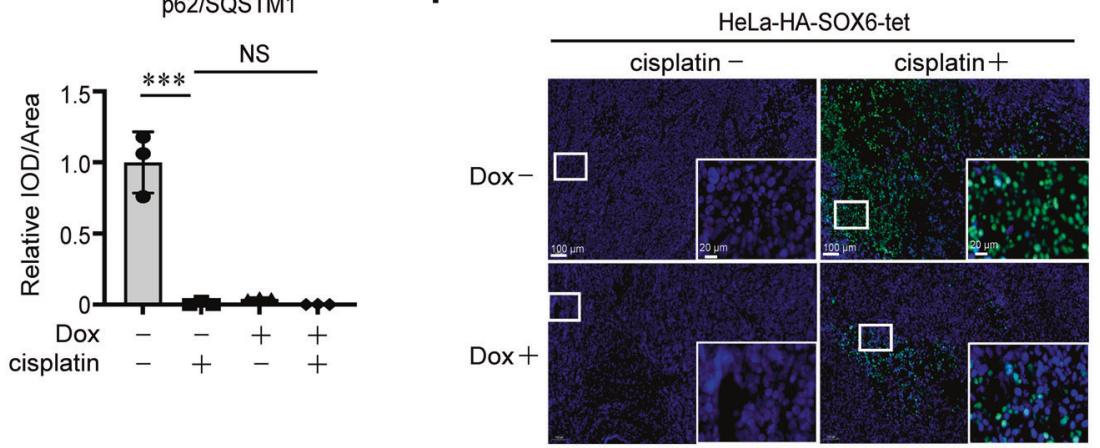

$\mathbf{F}$

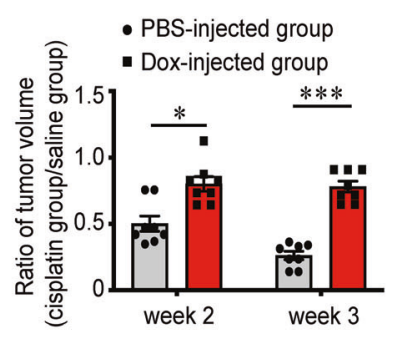

$0 \quad 50100150200250300$

B

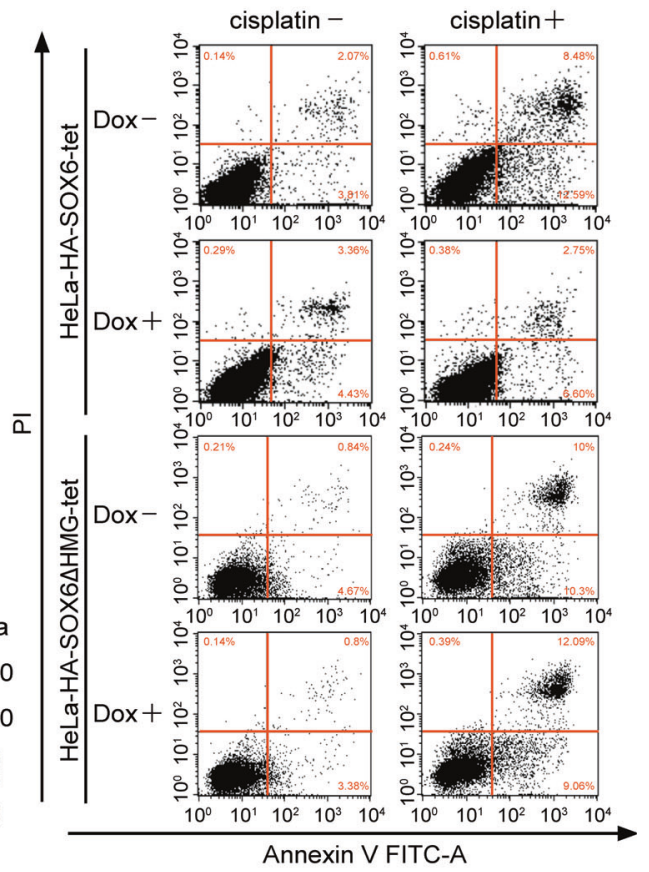

G

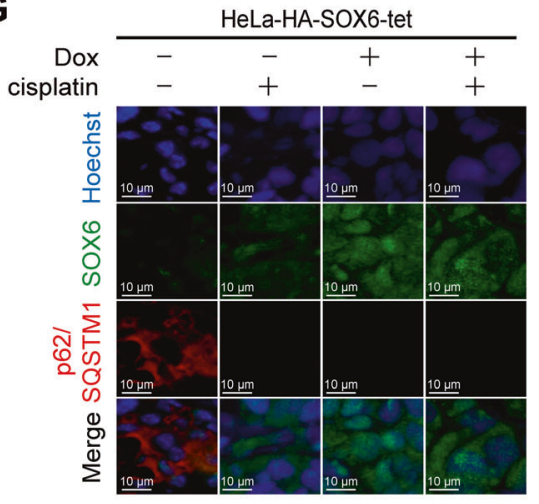

J

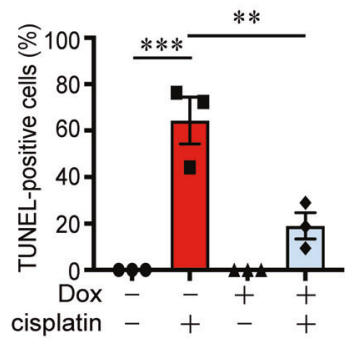

under PF-06260933 treatment (Fig. 7B). Taken together, these results suggested that the sensitivity of cervical cancer cells to cisplatin could be increased by inhibiting SOX6-induced autophagy through both autophagy and MAP4K4 inhibitors.

\section{DISCUSSION}

Cancer chemotherapy is curative in treating a wide range of tumors by inhibiting proliferation and inducing apoptosis of tumor cells $[44,45]$. As one of the platinum-based chemotherapeutic agents, cisplatin is widely used in the treatment of advanced cervical cancer, and it can exert anti-tumor function by inducing DNA damage, cell cycle arrest and apoptosis [40, 46]. However, high incidence of mortality is still observed due to the low sensitivity to chemotherapeutic agents or even drug resistance [47]. Therefore, it seems necessary to enhance the sensitivity of tumor cells to chemotherapeutic agents and search for the markers that can possibly predict the efficacy of 
Fig. 5 The effect of SOX6-induced autophagy in the sensitivity of cervical cancer cells to cisplatin treatment in vitro and in vivo. A Top 20 Gene Ontology (GO)-enriched terms of MAP4K4 gene. *indicated the terms related to apoptosis and cell death. B Flow cytometry analysis on the apoptosis of HeLa-HA-SOX6-tet and HeLa-HA-SOX6 $\Delta$ HMG-tet cells with or without cisplatin $(20 \mu \mathrm{M})$ treatment for $48 \mathrm{~h}$. C The percentage of apoptotic cells in flow cytometry analysis. D Western blotting analysis on the protein levels of PARP and caspase 9 in HeLa-HA-SOX6-tet cells with or without cisplatin $(20 \mu \mathrm{M})$ treatment. $\alpha$-tubulin protein was used as the internal control. E Experimental design of the steps used to produce xenograft in BALB/c nude mice. F Subcutaneously injection of the HeLa-HA-SOX6-tet cells into the left flank of nude mice, which were subsequently divided into two groups at 1-week post-injection and were intraperitoneally injected with Dox (20 mg/kg, PBS as solvent control) every day and cisplatin ( $3 \mathrm{mg} / \mathrm{kg}$, saline as solvent control) every other day for the next 2 weeks. The relative sizes of the tumors were represented by the ratio of the tumor size in cisplatin group to those in saline group. G Immunofluorescence staining of SOX6 (green) and p62/SQSTM1 protein (red) in the frozen section of xenograft tumor tissues. H Relative expression level of p62/SQSTM1 indicated by fluorescent density. IOD integral optical density. I Immunofluorescence staining of TUNEL (green) in the frozen section of xenograft tumor tissues. J The percentage of TUNEL-positive cells in the frozen section of xenograft tumor tissues. Data are mean \pm SEM of three independent experiments or at least three fields scored. $\left({ }^{*} P<0.05\right.$, ${ }^{* *} P<0.01,{ }^{* *} P<0.001$, NS non-significant, Student's $t$-test, two tails for $\mathbf{F}$, one-way ANOVA and post hoc Tukey tests for $\mathbf{C}, \mathbf{H}, \mathbf{J})$.

chemotherapy. Autophagy is a highly conserved process in eukaryotic cells and is regarded as a "double-edged sword" exerting protective or detrimental effects in tumorigenesis $[48,49]$.

In this study, we firstly found that SOX6-induced autophagy of cervical cancer cells depending on its HMG domain, and MAP4K4 gene was identified as the potential target gene of SOX6 through microarray and RNA-sequencing analyses. Next, two potential binding sites of SOX6 were identified at 430-444 and 59-77 bp upstream of the TSS of MAP4K4 gene, and SOX6 could enhance the transcriptional activity of MAP4K4 gene promoter by binding two binding sites with its HMG domain, suggesting that MAP4K4 gene was the target gene of SOX6. Further, we found that MAP4K4 not only could mediate the SOX6-induced autophagy, but also directly induce autophagy though activating MAPK/ERK pathway and inhibiting PI3K-Akt-mTOR pathway. Furthermore, SOX6 could significantly inhibit the cisplatin-induced apoptosis of cervical cancer cells dependent on its HMG domain in vitro and in vivo. In turn, the expression of endogenous SOX6 could be upregulated under cisplatin treatment. Based on the higher sensitivity of CaSki cells to cisplatin compared to HeLa cells, there was the possibility that the cisplatin-induced higher level of endogenous SOX6 might contribute to the lower sensitivity to cisplatin in HeLa cells. In line with this possibility, the sensitivity of HeLa cells to cisplatin was increased when the endogenous SOX6 was knocked out, thus the level of SOX6 might be able to predict the efficacy of cisplatin chemotherapy to cervical cancer cells. Moreover, the role of SOX6induced autophagy was explored in cervical cancer patients who underwent routine neoadjuvant chemotherapy. For the patients who were sensitive to chemotherapy, the levels of SOX6 and its downstream MAP4K4 proteins were lower, but the levels of p62/ SQSTM1 were higher than those in patients who were not sensitive to cisplatin, which further confirmed that the high level of SOX6-induced autophagy mediated by MAP4K4 might reduce the sensitivity of cisplatin chemotherapy.

Finally, we further investigated how to increase the sensitivity of cervical cancer cells to cisplatin based on the existing compounds targeting the pathways of SOX6-induced autophagy. Firstly, the SOX6-reduced sensitivity to cisplatin could be turned over under the treatment of Baf A1, an autophagy-specific inhibitor. Further, the sensitivity to cisplatin could also be increased by inhibiting the expression of endogenous MAP4K4 under the treatment of PF06260933, an MAP4K4-specific inhibitor. Taken together, the sensitivity of cervical cancer cells to cisplatin could be increased by inhibiting the SOX6-induced autophagy through both autophagy and MAP4K4 inhibitors. Since MAP4K4 was at the upstream of SOX6-induced autophagy, its inhibitor might be more specific in inhibiting autophagy and subsequently increasing the sensitivity of cervical cancer cells to cisplatin chemotherapy.

In conclusion, SOX6 could transactivate its direct target gene MAP4K4 through binding to its specific binding sites located at the promoter of MAP4K4 gene, then autophagy was induced by the MAP4K4-mediated activation of MAPK/ERK pathway and inhibition of PI3K-Akt-mTOR pathway. Further, the sensitivity to cisplatin treatment could be reduced by the SOX6-induced autophagy, and the endogenous SOX6 upregulated by cisplatin might contribute to the reduced sensitivity to cisplatin treatment. Moreover, the sensitivity of cervical cancer cells to cisplatin could be increased by inhibiting SOX6-induced autophagy through both autophagy and MAP4K4 inhibitors (Fig. 7C). This study uncovered the mechanism of SOX6-induced autophagy in cervical cancer cells and its role in regulation of the sensitivity to cisplatin chemotherapy, which might explain the underlying reason for the poor response of partial cervical cancer patients to the platinum-based chemotherapy. More importantly, this study shed new light on the usage of MAP4K4 inhibitors to increase the sensitivity of cervical cancer cells to the platinum-based chemotherapy.

\section{MATERIALS AND METHODS \\ Cell lines}

Human cervical cancer cell lines HeLa, CaSki and SiHa were used and were maintained in Dulbecco's modified Eagle medium (DMEM) (Corning, Corning, New York, USA) supplemented with $10 \%$ fetal bovine serum (FBS) (PAN, Adenbach, Bavaria, Germany) in this study. HeLa-HA-SOX6-tet and HeLa-HA-SOX6 $\triangle$ HMG-tet cell lines were constructed previously in our laboratory and were maintained in DMEM (Corning, Corning, New York, USA) supplemented with $10 \%$ FBS (PAN, Adenbach, Bavaria, Germany), $400 \mu \mathrm{g} / \mathrm{mL}$ G418 (Amresco, Solon, Washington, USA) and $4 \mu \mathrm{g} / \mathrm{mL}$ blasticidin (Thermo Fisher Scientific, Waltham, Massachusetts, USA). Final concentration of $4 \mu \mathrm{g} / \mathrm{mL}$ of doxycycline (Merck, Kenilworth, New Jersey, USA) was added into the culture medium of HeLa-SOX6-tet and HeLa-

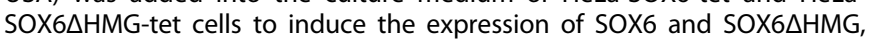
respectively.

HeLa cells with endogenous SOX6 gene knockout (HeLa-SOX6KO) were constructed in our laboratory. Specifically, HeLa cells were seeded into sixwell plates and transduced with lentivirus that was produced by HEK 293FT cells transfected with lenti-CRISPR-SOX6 plasmids, and together with $10 \mu \mathrm{g} / \mathrm{mL}$ polybrene (Santa Cruz Biotechnology, Santa Cruz, CA, USA). After $48 \mathrm{~h}$ of infection, the infected HeLa cells were maintained in DMEM supplemented with $10 \%$ FBS and $2 \mu \mathrm{g} / \mathrm{mL}$ puromycin (Thermo Fisher Scientific, Waltham, Massachusetts, USA) for another $48 \mathrm{~h}$, and then 100 cells were counted and subsequently seeded into a 96-well plate. Several single clones of HeLa cells were subsequently identified by sequencing of PCR product and western blotting to establish HeLa cells with endogenous SOX6 gene knockout. All cell lines used in our research were tested negative for mycoplasma contamination.

\section{Cervical cancer tumor samples}

Total 14 cervical cancer patients from the Gynecology Department of the Third Hospital of Peking University were included in our research, all of whom were diagnosed to be at local advanced stage and had received routine treatment by cisplatin combined with paclitaxel for 2 courses. The cervical cancer tissues from 14 cervical cancer patients were collected, half of whom were sensitive to chemotherapy according to the new guidelines to evaluate the response to treatment in solid tumors. This study was 
approved by the Ethics Committee of Third Hospital of Peking University. Written informed consent was obtained from each patient.

\section{Plasmids}

The plex-HA-SOX6, plex-HA-SOX6 $\triangle \mathrm{HMG,} \mathrm{GFP-LC3,} \mathrm{and} \mathrm{PRL-TK} \mathrm{plasmids}$ were constructed previously and maintained in our laboratory. The pGL3-
MAP4K4-promoter was constructed by inserting the promoter sequence of MAP4K4 gene into pGL3-basic plasmid. Specifically, the promoter sequence of MAP4K4 gene was amplified by PCR from HeLa DNA, and the PCR products were subsequently ligated into the pGL3-basic plasmid after digestion with Kpn I and Hind III. The pGL3-MAP4K4-promoter mutant 1 and pGL3-MAP4K4-promoter mutant 2 were constructed on the basis of pGL3-MAP4K4-promoter plasmid by the site directed rapid mutagenesis
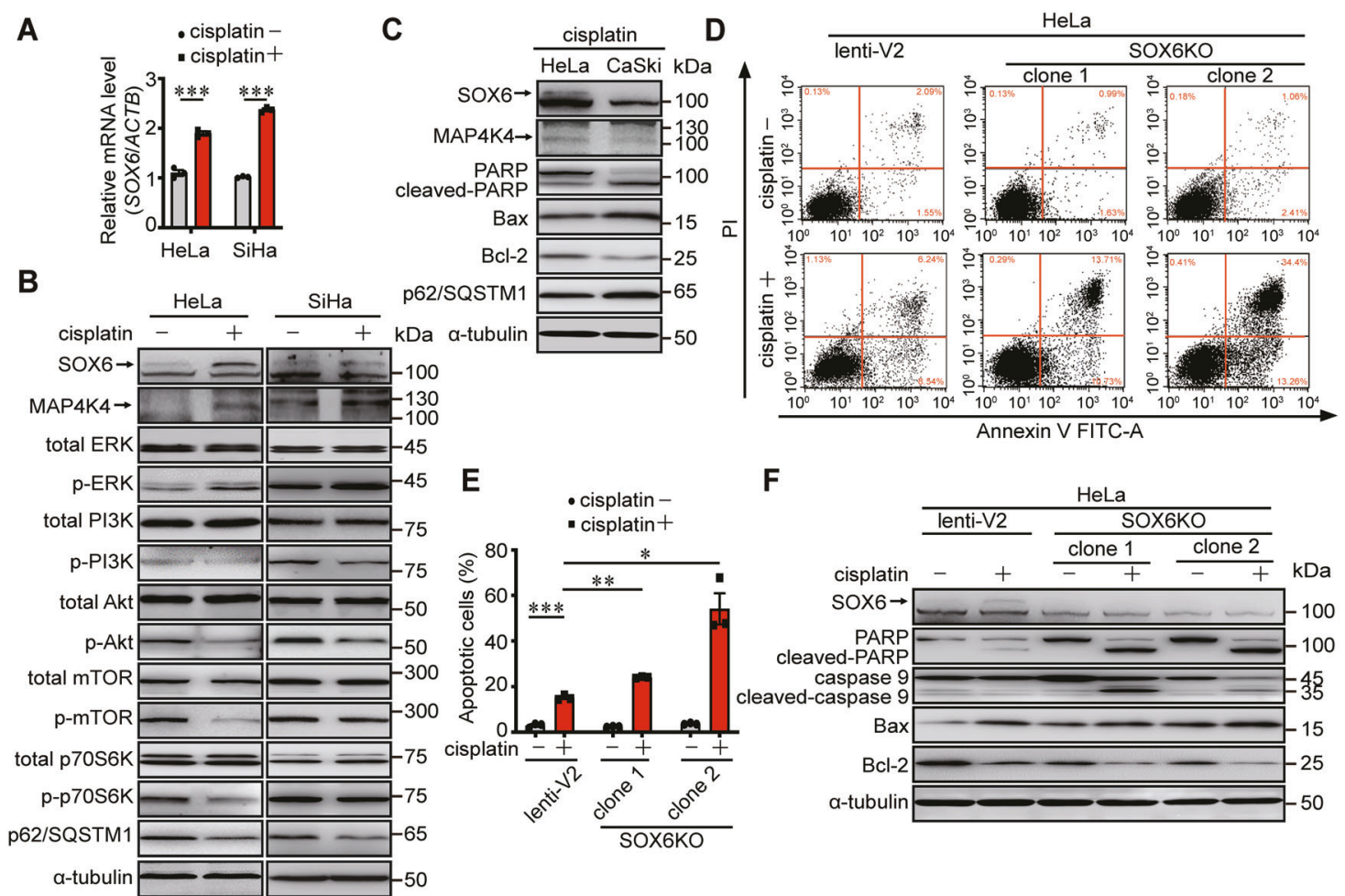

G
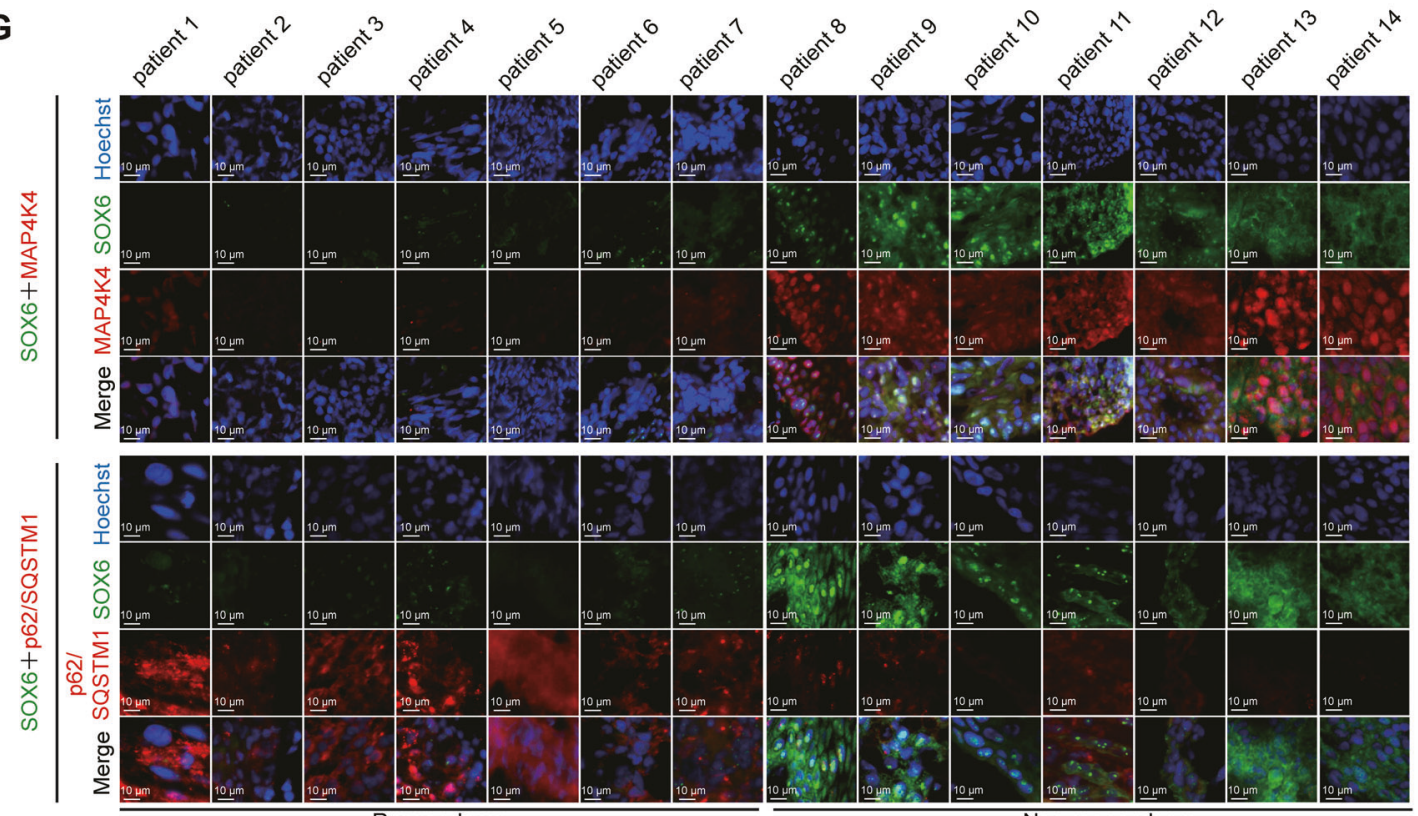

H

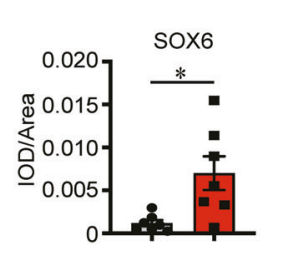

I

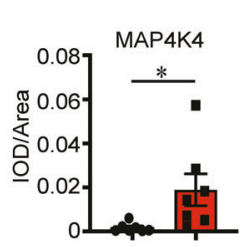

$\mathbf{J}$

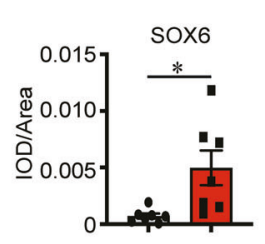

K

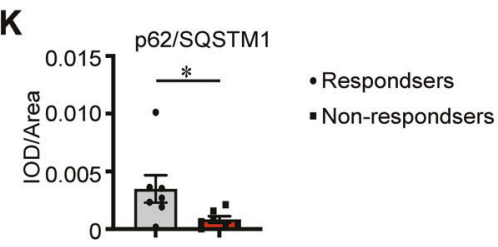


Fig. 6 The effect of endogenous SOX6 and MAP4K4 in the sensitivity to cisplatin treatment. A The RT-qPCR (SYBR Green) analysis on the levels of SOX6 mRNA in HeLa and SiHa cells that were treated with or without cisplatin $(20 \mu \mathrm{M})$. $\beta$-Actin (ACTB) mRNA was used as the internal control. B Western blotting analysis on the protein levels of key molecules within MAPK/ERK and PI3K-Akt-mTOR signaling pathway in HeLa and $\mathrm{SiHa}$ cells that were treated with or without cisplatin $(20 \mu \mathrm{M})$. $\alpha$-tubulin protein was used as the internal control. C Western blotting analysis on the protein levels of SOX6, MAP4K4, PARP, Bax, Bcl-2, and p62/SQSTM1 in HeLa and CaSki cells treated with or without cisplatin $(20 \mu \mathrm{M})$ treatment. D Flow cytometry analysis of apoptosis in two clones of HeLa-SOX6KO cells and the control HeLa cells (lenti-V2) with or without cisplatin $(20 \mu \mathrm{M})$ treatment. E The percentage of apoptotic cells in flow cytometry analysis. $\mathbf{F}$ Western blotting analysis on the protein levels of PARP, caspase 9, Bax, and Bcl-2 in two clones of HeLa-SOX6KO cells and the control HeLa cells (lenti-V2) cells with or without cisplatin $(20 \mu \mathrm{M})$ treatment. G Representative immunofluorescent staining of SOX6 (green) and MAP4K4 (red) or p62/SQSTM1 (red) in samples collected from 14 cervical cancer patients with routine cisplatin treatment. H Relative expression level of SOX6 indicated by fluorescent density. I Relative expression level of MAP4K4 indicated by fluorescent density. J Relative expression level of SOX6 indicated by fluorescent density. K Relative expression level of p62/SQSTM1 indicated by fluorescent density. Data are the mean \pm SEM of three independent experiments or at least three fields scored $\left({ }^{*} P<0.05,{ }^{*} P<0.01,{ }^{* *} P<0.001\right.$, Student's $t$-test, two tails for $\mathbf{A}$, E, one-way ANOVA and post hoc Tukey tests for $\left.\mathbf{H}-\mathbf{K}\right)$. IOD, integral optical density.

A

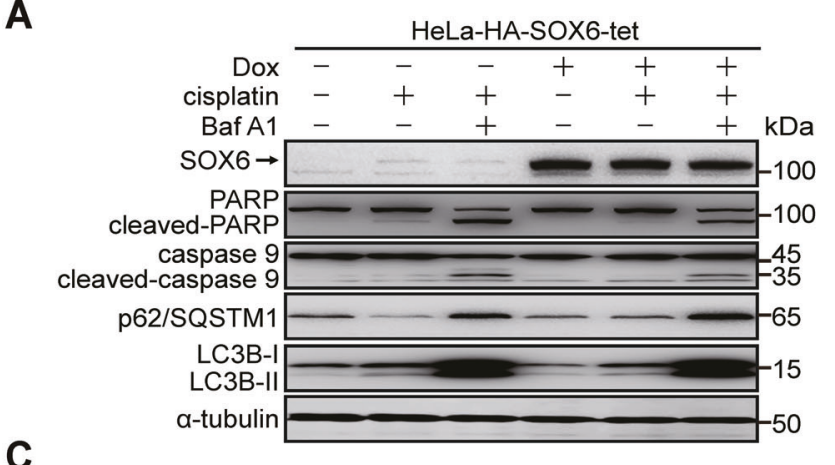

C
B

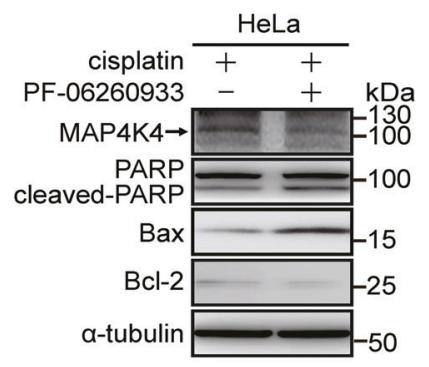

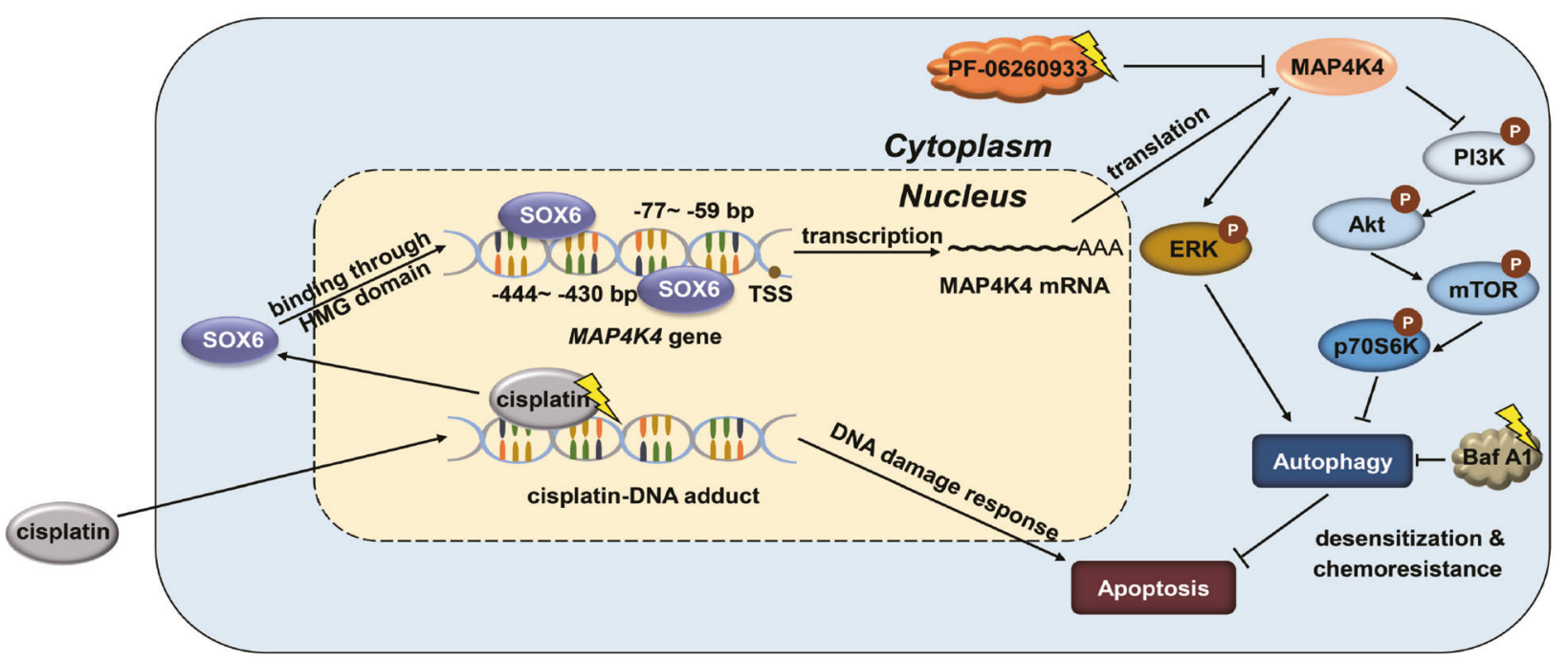

Fig. 7 The effects of inhibiting autophagy and MAP4K4 expression in the sensitivity of cervical cancer cells to cisplatin treatment. A Western blotting analysis on the protein levels of SOX6, PARP, caspase 9, p62/SQSTM1 and LC3B in HeLa-HA-SOX6-tet cells treated with or without Dox $(4 \mu \mathrm{g} / \mathrm{mL})$, cisplatin $(20 \mu \mathrm{M})$ for $48 \mathrm{~h}$ and/or Baf $\mathrm{A} 1(10 \mathrm{nM})$ for the last $6 \mathrm{~h}$. $\alpha$-tubulin protein was used as the internal control. B Western blotting analysis on the protein levels of MAP4K4, PARP, Bax, and Bcl-2 in HeLa cells under cisplatin ( $20 \mu \mathrm{M})$ treatment and treated with or without PF-06260933 $(3 \mu \mathrm{M})$. C The graphical abstract on the mechanism and clinical significance of SOX6-induced autophagy in cervical cancer cells.

methods. The lenti-CRISPR-SOX6 plasmid was constructed by ligating the annealed oligonucleotides of SOX6 gene-specific gRNA into the lentiCRISPRv2 plasmid digested with Bsm BI. The $\mathrm{pCDH}-3 \times$ flag-MAP4K4 plasmid was constructed by ligating the synthesized sequence of the MAP4K4 CDS sequence containing $3 \times$ flag tag sequence into the $\mathrm{pCDH}$-vector plasmid after digestion with $\mathrm{Bam} \mathrm{HI}$ and $\mathrm{Xba} \mathrm{I}$. The primer sequences used for plasmid construction were listed in Supplementary Table S1.

\section{Antibodies and reagents}

The following primary antibodies were purchased from Abcam: anti-SOX6 (ab30455), anti-p62/SQSTM1 (ab109012). The anti-HA-tag (M180-3) antibody was purchased from MBL International. The following antibodies were purchased from Cell Signaling Technology: anti-LC3B (2775 S), antip44/42 MAPK (9102 S), anti-phospho-p44/42 MAPK (4370 S), anti-Akt (2920 S), anti-phospho- Akt (4060 S), anti-mTOR (2983 S), anti-phosphomTOR ( $5536 \mathrm{~S})$, anti-p70 S6 kinase (2708 S), anti-phospho-p70 S6 kinase (9205 S), anti-PARP (9542), anti-caspase 9 (9502), anti-Bax (5023) and anti$\mathrm{BCl}-2$ (15071). The following antibodies were purchased from Santa Cruz Biotechnology: anti-MAP4K4 (sc-100445) and anti-phospho-p38 (sc166182). Second antibodies were IRDye 680LT donkey anti-rabbit lgG (LICOR, 926-68023), IRDye 800CW donkey anti-mouse IgG (LICOR, 92632212), HRP-linked anti-rabbit IgG (Cell Signaling technology, 7074 S) and HRP-linked anti-mouse IgG (Cell Signaling technology, 7076S). The 
antibodies mentioned above were all used for western blotting analyses. The following antibodies were used in immunofluorescence: anti-SOX6 (Abcam, ab30455), anti-p62/SQSTM1 (Cell Signaling technology, 88588 S), FITC-conjugated goat anti-mouse IgG (Zhongshan Golden Bridge, ZF0312), FITC-conjugated goat anti-rabbit IgG (Zhongshan Golden Bridge, ZF0311), TRITC-conjugated goat anti-mouse IgG (Zhongshan Golden Bridge, ZF-0313) and TRITC-conjugated goat anti-rabbit IgG (Zhongshan Golden Bridge, ZF-0316). The following antibodies were used in ChIP: anti-HA-tag (Abcam, ab9110) and IgG from rabbit serum (Sigma-Aldrich, 15006). Other regents used in this study were: bafilomycin A1 (Baf A1; Santa Cruz Biotechnology, sc-201550), PF-06260933 (TargetMol, T4221) and cisplatin (Pharmabiology, C21384).

\section{Microarray analysis}

Total RNA was extracted by TRIzol ${ }^{\oplus}$ Reagent (Thermo Fisher Scientific, Waltham, Massachusetts, USA) according to the manufacturer's instructions. After identification of the purity and integrity, RNA samples were sent for microarray analysis carried out by Shanghai Biotechnology Corporation. Data were analyzed by the online SBC analysis system (SAS; http://sas.ebioservice.com). KEGG signaling pathway enrichment analysis and gene ontology (GO) enrichment analysis were carried out by the DAVID (http://david.abcc.ncifcrf.gov).

\section{RNA-sequencing analysis}

Total RNA was extracted from the tissue using TRIzol ${ }^{\circledR}$ Reagent (Thermo Fisher Scientific, Waltham, Massachusetts, USA) according to the manufacturer's instructions, and the genomic DNA was removed using DNase I (TaKara, Akishima, Tokyo, Japan). Then RNA quality was determined by 2100 Bioanalyser (Agilent) and quantified using the ND-2000 (Thermo Fisher Scientific, Waltham, Massachusetts, USA). Only high-quality RNA sample $(\mathrm{OD} 260 / 280=1.8-2.2, \mathrm{OD} 260 / 230 \geq 2.0, \mathrm{RIN} \geq 6.5,28 \mathrm{~S}: 18 \mathrm{~S} \geq 1.0$, $>2 \mu \mathrm{g}$ ) was used to construct sequencing library and sequencing carried out by Majorbio corporation. Functional enrichment analysis including KEGG and GO were analyzed on the free online platform of Majorbio Cloud Platform (www.majorbio.com). The raw RNA-sequencing data have been deposited in the Genome Sequence Archive in National Genomics Data Center, China National Center for Bioinformation under the accession number of HRA001566.

\section{RNA interference}

HeLa-SOX6-tet cells were transfected with MAP4K4-specific siRNA oligonucleotide duplex (RiBobio, Guangzhou, Guangdong, China) using Lipofectamine RNAiMAX (Thermo Fisher Scientific, Waltham, Massachusetts, USA). Briefly, siRNA and Lipofectamine RNAiMAX were added into Opti-MEM medium (Thermo Fisher Scientific, Waltham, Massachusetts, USA) in turn, then mixed them gently and incubated for $20 \mathrm{~min}$ at room temperature. Next, the mixture was added to the culture medium at a final concentration of $50 \mathrm{nM}$ siRNA. The effectiveness of siRNA knockdown was evaluated by reverse-transcription and quantitative polymerase chain reaction (RT-qPCR) and western blotting at 3 days post transfection.

\section{RNA extraction, reverse transcription, and qPCR}

Total RNA was extracted using TRIzol ${ }^{\circ}$ Reagent (Thermo Fisher Scientific, Waltham, Massachusetts, USA) according to the manufacturer's instructions. Reverse transcription was performed using Transcriptor First Strand cDNA Synthesis Kit (Roche, Basel, Kanton Basel, Switzerland). The primer used for reverse transcription was random primer. The mRNA levels of MAP4K4, SOX6, and ACTB were detected by QPCR (SYBR Green method) using an Applied Biosystems StepOne plus Real-Time PCR system (Thermo Fisher Scientific, Waltham, Massachusetts, USA). The primers used to detect the mRNA levels of MAP4K4, SOX6 and ACTB were listed in Supplementary Table S2.

\section{Chromatin immunoprecipitation assay (ChIP)}

The ChIP assay was performed using Simple ChIP Enzymatic chromatin IP kit (Cell Signaling Technology, Danvers, Massachusetts, USA) as previously described [50]. Briefly, HeLa cells were transfected with plex-HA-SOX6 or plex-HA-SOX6 $\triangle \mathrm{HMG}$ plasmid. At $48 \mathrm{~h}$ post transfection, cells were treated with $1 \%$ methanol at room temperature for $10 \mathrm{~min}$, and then added glycine to stop the reaction. After washing with cold PBS for three times, cells were lysed with SDS lysis buffer, and then the lysates were fragmented by ultrasound to get $300-500 \mathrm{bp}$ DNA fragments in length.
Finally, the lysates were incubated with rabbit anti-HA-tag-ChIP grade (Abcam, ab9110) or lgG from rabbit serum (Sigma-Aldrich, 15006).

\section{Dual-luciferase reporter assay}

At $48 \mathrm{~h}$ post transfection, cells were washed in PBS and lysed with passive lysate buffer (Promega, Madison, Wisconsin, USA), and then the relative luciferase activity was measured using dual-luciferase assay kit (Promega, Madison, Wisconsin, USA) following the manufacturer's instructions.

\section{Xenograft implantation in vivo}

HeLa-HA-SOX6-tet and HeLa-HA-SOX6AHMG-tet cells were subcutaneously injected into the left flank of $12 \mathrm{BALB} / \mathrm{c}$ female nude mice $\left(3 \times 10^{6}\right.$ cells for each mouse), respectively. Subsequently, these mice were randomly divided into two groups, one group of them (12 mice) were daily intraperitoneally injected with Dox $(20 \mathrm{mg} / \mathrm{kg})$, and the other group (12 mice) were daily intraperitoneally injected with solvent control-phosphate buffered solution (PBS) for 3 weeks, and the tumor blocks were collected when the mice were sacrificed under anesthesia at 3 weeks post-injection.

For the investigation of influence of the SOX6-induced autophagy on the sensitivity to cervical cancer cells to cisplatin treatment, HeLa-HASOX6-tet cells were subcutaneously injected into the left flank of $32 \mathrm{BALB} / \mathrm{c}$ female nude mice $\left(3 \times 10^{6}\right.$ cells for each mouse), then the mice were randomly divided into four groups (8 mice for each group) at 1-week postinjection and were daily intraperitoneally injected with Dox $(20 \mathrm{mg} / \mathrm{kg}, \mathrm{PBS}$ as solvent control) every day and cisplatin $(3 \mathrm{mg} / \mathrm{kg}$, saline as solvent control) every other day for the next 2 weeks. The sizes of tumors in mice were measured every week, and the tumor blocks were collected when the mice were sacrificed under anesthesia at 3 weeks post-injection. This study was approved by the Ethics Committee of Peking University Health Science Center.

\section{Western blotting}

Cells were harvested and lysed in RIPA lysis buffer, and the lysate was subsequently subjected to polyacrylamide gel electrophoresis. The proteins in polyacrylamide gel were then transferred onto a PVDF membrane by the method of wet electrophoretic transfer. The membrane was then blocked in 5\% skimmed milk for $1 \mathrm{~h}$ and incubated with the primary antibodies at $4{ }^{\circ} \mathrm{C}$ overnight. After washing in TBST for 3 times, the membrane was incubated with the secondary antibodies for $1 \mathrm{~h}$ at room temperature. Then the levels of proteins were detected by Odessey infrared imaging system (LICOR, Lincoln, Nebraska, USA) or chemiluminescence imaging analysis system (Tanon, Shanghai, China).

\section{Immunofluorescence staining}

Cells were washed with cold PBS for 3 times and were fixed with 4\% paraformaldehyde at room temperature for $20 \mathrm{~min}$, followed by permeabilized with $0.5 \%$ Triton X-100 at room temperature for $15 \mathrm{~min}$. Then cells were blocked in $20 \%$ goat serum at room temperature for $1 \mathrm{~h}$ and incubated with the primary antibody at $4{ }^{\circ} \mathrm{C}$ overnight. After washed with PBS for 3 times, cells were then incubated with the secondary antibodies at room temperature for $1 \mathrm{~h}$, followed by staining with Hoechst 33342. At last, cells were examined under a TCS-SP8 STED confocal laser scanning microscope (Leica, Frankfurt, Hesse-Darmstadt, Germany) or an inverted fluorescent microscope (Leica, Frankfurt, Hesse-Darmstadt, Germany).

The immunofluorescent staining was double-blinded and carried out by Wuhan Servicebio technology cooperation (Wuhan, Hubei, China). The tissue samples of the xenograft and the cervical cancer patients were solidified in liquid nitrogen, and were sent for the immunofluorescent staining. The areal density (IOD/Area) was calculated by image-pro plus 6.0 software (Media Cybernetics, Inc., Rockville, MD, USA) to reflect the relative protein level.

\section{Apoptosis assay}

Apoptotic cells were detected using Annexin V-FITC/PI apoptosis detection kit (Dojindo, Kumamoto, Kyushu, Japan) according to the manufacturer's instructions. The early apoptotic cells were stained with Annexin V-FITC, and the late apoptotic cells were stained with propidium iodide (PI). The percentage of apoptotic cells were analyzed and calculated with Flowjo software (v7.6, Stanford, California, USA). The cell apoptosis in xenograft was detected by Terminal deoxynucleotidyl transferase (TdT) dUTP NickEnd Labeling (TUNEL) assay on frozen sections, which were carried out by Wuhan Servicebio technology cooperation. 


\section{Transmission electron microscopy}

Cells were harvested and fixed with $2.5 \%$ glutaraldehyde followed by stained with $1 \%$ osmic acid. Next, cells were dehydrated with different concentrations of acetone, and then were embed and stained with lead citrate and uranyl acetate. Autophagic structures were then detected using a JEM-1400 transmission electron microscope (JEOL, Akishima, Tokyo, Japan).

\section{Statistical analysis}

All data were presented as mean \pm standard error of mean (SEM). Statistical analysis was performed using GraphPad prism 8.0 software (GraphPad Software, San Diego, California, USA). Comparisons among groups were analyzed by one-way ANOVA and post hoc Tukey tests, and the differences between groups were analyzed by two-tailed Student's $t$-tests, and a $P$ value less than 0.05 was considered statistically significant.

\section{DATA AVAILABILITY}

All data needed to evaluate the conclusions in the paper are present in the paper and/or the Supplementary Materials. Additional data related to this paper are available on reasonable request.

\section{REFERENCES}

1. Sinclair A, Berta P, Palmer MS, Hawkins JR, Griffiths BL, Smith MJ, et al. A gene from the human sex-determining region encodes a protein with homology to a conserved DNA-binding motif. Nature. 1990;346:240-4.

2. Gubbay J, Collignon J, Koopman P, Capel B, Economou A, Münsterberg A, et al. A gene mapping to the sex-determining region of the mouse $Y$ chromosome is a member of a novel family of embryonically expressed genes. Nature. 1990:346:245-50.

3. Sarkar A, Hochedlinger K. The sox family of transcription factors: versatile regulators of stem and progenitor cell fate. Cell Stem Cell. 2013;12:15-30.

4. Kamachi $\mathrm{Y}$, Kondoh $\mathrm{H}$. Sox proteins: regulators of cell fate specification and differentiation. Development. 2013;140:4129-44.

5. Wang Z, Li J, Li K, Xu J. SOX6 is downregulated in osteosarcoma and suppresses the migration, invasion and epithelial-mesenchymal transition via TWIST1 regulation. Mol Med Rep. 2018;17:6803-11.

6. Bowles J, Schepers G, Koopman P. Phylogeny of the SOX family of developmental transcription factors based on sequence and structural indicators. Dev Biol. 2000;227:239-55.

7. Qin $\mathrm{Y}$, Tang $\mathrm{H}$, Xie F, Liu H, Zhu Y, Ai J, et al. Characterization of tumor-suppressive function of SOX6 in human esophageal squamous cell carcinoma. Clin Cancer Res. 2011;17:46-55.

8. Ueda R, Tsukada KO, Fusaki N, Soeda A, Kawase T, Kawakami Y, et al. Identification of HLA-A2- and A24-restricted T-cell epitopes derived from SOX6 expressed in glioma stem cells for immunotherapy. Int J Cancer. 2010;126:919-29.

9. Xie $Q$, Chen X, Lu F, Zhang T, Hao M, Wang $Y$, et al. Aberrant expression of microRNA 155 may accelerate cell proliferation by targeting sex-determining region $Y$ box 6 in hepatocellular carcinoma. Cancer. 2012;118:2431-42.

10. Guo X, Yang M, Gu H, Zhao J, Zou L. Decreased expression of SOX6 confers a poor prognosis in hepatocellular carcinoma. Cancer Epidemiol. 2013;37:732-6.

11. Cantu C, lerardi R, Alborelli I, Fugazza C, Cassinelli L, Piconese S, et al. Sox6 enhances erythroid differentiation in human erythroid progenitors. Blood. 2011;117:3669-79.

12. Iguchi $H$, Urashima $Y$, Inagaki $Y$, Ikeda $Y$, Okamura $M$, Tanaka $T$, et al. SOX6 suppresses cyclin D1 promoter activity by interacting with beta-catenin and histone deacetylase 1 , and its down-regulation induces pancreatic beta-cell proliferation. J Biol Chem. 2007;282:19052-61.

13. Lin M, Lei T, Zheng J, Chen S, Du L, Xie H. UBE2S mediates tumor progression via SOX6/beta-Catenin signaling in endometrial cancer. Int J Biochem Cell Biol. 2019:109:17-22.

14. Wang J, Ding S, Duan Z, Xie Q, Zhang T, Zhang X, et al. Role of p14ARF-HDM2-p53 axis in SOX6-mediated tumor suppression. Oncogene. 2016;35:1692-702.

15. World Health Organization. Human papillomavirus (HPV) and cervical cancer. 2020. https://www.who.int/mediacentre/factsheets/fs380/en/.

16. Real NE, Castro GN, Cuello-Carrión FD, Perinetti C, Röhrich $\mathrm{H}$, Cayado-Gutiérrez $\mathrm{N}$, et al. Molecular markers of DNA damage and repair in cervical cancer patients treated with cisplatin neoadjuvant chemotherapy: an exploratory study. Cell Stress Chaperones. 2017;22:811-22.

17. Al-Mansour Z, Verschraegen C. Locally advanced cervical cancer: what is the standard of care? Curr Opin Oncol. 2010;22:503-12.

18. Narayan S, Sharma N, Kapoor A, Sharma R, Kumar N, Singhal M, et al. Pros and Cons of Adding of Neoadjuvant Chemotherapy to Standard Concurrent
Chemoradiotherapy in Cervical Cancer: A Regional Cancer Center Experience. J Obstet Gynaecol India. 2016;66:385-90.

19. Guo J, Chen M, Ai G, Mao W, Li H, Zhou J. Hsa_circ_0023404 enhances cervical cancer metastasis and chemoresistance through VEGFA and autophagy signaling by sponging miR-5047. Biomed Pharmacother. 2019;115:108957.

20. Ravanan P, Srikumar IF, Talwar P. Autophagy: The spotlight for cellular stress responses. Life Sci. 2017;188:53-67.

21. Gozuacik D, Kimchi A. Autophagy as a cell death and tumor suppressor mechanism. Oncogene. 2004;23:2891-906.

22. Vanzo R, Bartkova J, Merchut-Maya JM, Hall A, Bouchal J, Dyrskjøt L, et al. Autophagy role(s) in response to oncogenes and DNA replication stress. Cell Death Differ. 2020;27:1134-53.

23. Chen $\mathrm{C}$, Lu L, Yan S, Yi H, Yao H, Wu D, et al. Autophagy and doxorubicin resistance in cancer. Anticancer Drugs. 2018;29:1-9.

24. Li Y, Lei Y, Yao N, Wang C, Hu N, Ye W, et al. Autophagy and multidrug resistance in cancer. Chin J Cancer. 2017;36:52.

25. Delpire E. The mammalian family of sterile 20p-like protein kinases. Pflug Arch. 2009;458:953-67.

26. Xue Y, Wang X, Li Z, Gotoh N, Chapman D, Skolnik EY. Mesodermal patterning defect in mice lacking the Ste20 NCK interacting kinase (NIK). Development. 2001;128:1559-72.

27. Vitorino P, Yeung S, Crow A, Bakke J, Smyczek T, West K, et al. MAP4K4 regulates integrin-FERM binding to control endothelial cell motility. Nature. 2015;519:425-30.

28. Aouadi M, Tesz GJ, Nicoloro SM, Wang M, Chouinard M, Soto E, et al. Orally delivered siRNA targeting macrophage Map4k4 suppresses systemic inflammation. Nature. 2009;458:1180-4.

29. Chuang $H$, Sheu WH, Lin Y, Tsai $C$, Yang $C$, Cheng $Y$, et al. HGK/MAP4K4 deficiency induces TRAF2 stabilization and Th17 differentiation leading to insulin resistance. Nat Commun. 2014;5:4602.

30. Roth Flach RJ, Skoura A, Matevossian A, Danai LV, Zheng W, Cortes C, et al Endothelial protein kinase MAP4K4 promotes vascular inflammation and atherosclerosis. Nat Commun. 2015;6:8995.

31. Wright JH, Wang X, Manning G, LaMere BJ, Le P, Zhu S, et al. The STE20 kinase HGK is broadly expressed in human tumor cells and can modulate cellular transformation, invasion, and adhesion. Mol Cell Biol. 2003;23:2068-82.

32. Liu A, Cai J, Zhao X, Jiang T, He T, Fu H, et al. ShRNA-targeted MAP4K4 inhibits hepatocellular carcinoma growth. Clin Cancer Res. 2011;17:710-20.

33. Qiu M, Qian Y, Zhao X, Wang S, Feng X, Chen X, et al. Expression and prognostic significance of MAP4K4 in lung adenocarcinoma. Pathol Res Pr. 2012;208:541-8.

34. Rizzardi AE, Rosener NK, Koopmeiners JS, Vogel RI, Metzger GJ, Forster CL, et al Evaluation of protein biomarkers of prostate cancer aggressiveness. BMC Cancer. 2014;14:244.

35. Pankiv S, Clausen TH, Lamark T, Andreas B, Bruun JA, Outzen H, et al. p62/ SQSTM1 binds directly to Atg8/LC3 to facilitate degradation of ubiquitinated protein aggregates by autophagy. J Biol Chem. 2007;282:24131-45.

36. Gao X, Gao C, Liu G, Hu J. MAP4K4: an emerging therapeutic target in cancer. Cell Biosci. 2016;6:56.

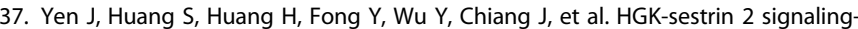
mediated autophagy contributes to antitumor efficacy of Tanshinone IIA in human osteosarcoma cells. Cell Death Dis. 2018;9:1003.

38. Wei $R$, Xiao $Y$, Song $Y$, Yuan $H$, Luo J, Xu W. FAT4 regulates the EMT and autophagy in colorectal cancer cells in part via the PI3K-AKT signaling axis. J Exp Clin Cancer Res. 2019;38:112.

39. Wu M, Ma Y, Chen X, Liang N, Qu S, Chen H. Hyperuricemia causes kidney damage by promoting autophagy and NLRP3-mediated inflammation in rats with urate oxidase deficiency. Dis Model Mech. 2021;14:dmm048041.

40. Yimit A, Adebali O, Sancar A, Jiang Y. Differential damage and repair of DNAadducts induced by anti-cancer drug cisplatin across mouse organs. Nat Commun. 2019;10:309.

41. Zhang B, Cao K, Liu Z, Shan W, Wen Q, Wang R. Receptor interacting protein kinase 3 promotes cisplatin-induced necroptosis in apoptosis-resistant HepG2/ DDP cells. Neoplasma. 2019;66:694-703.

42. Zhou F, Yang X, Zhao H, Liu Y, Feng Y, An R, et al. Down-regulation of OGT promotes cisplatin resistance by inducing autophagy in ovarian cancer. Theranostics. 2018;8:5200-12.

43. Duffaud $F$, Therasse $P$. New guidelines to evaluate the response to treatment in solid tumors. Bull Cancer. 2000;87:881-6.

44. Vengoji R, Macha MA, Nimmakayala RK, Rachagani S, Siddiqui JA, Mallya K, et al. Afatinib and Temozolomide combination inhibits tumorigenesis by targeting EGFRvIll-cMet signaling in glioblastoma cells. J Exp Clin Cancer Res. 2019;38:266.

45. Liu L, Fan J, Ai G, Liu J, Luo N, Li C, et al. Berberine in combination with cisplatin induces necroptosis and apoptosis in ovarian cancer cells. Biol Res. 2019:52:37. 
14

46. Plaimee P, Weerapreeyakul N, Barusrux S, Johns NP. Melatonin potentiates cisplatin-induced apoptosis and cell cycle arrest in human lung adenocarcinoma cells. Cell Prolif. 2015;48:67-77.

47. He L, Luo L, Zhu H, Yang $H$, Zhang $Y$, Wu H, et al. FEN1 promotes tumor progression and confers cisplatin resistance in non-small-cell lung cancer. Mol Oncol. 2017;11:640-54.

48. Vander Heiden MG, Cantley LC, Thompson CB. Understanding the Warburg effect: the metabolic requirements of cell proliferation. Science. 2009;324:1029-33.

49. Takamura A, Komatsu M, Hara T, Sakamoto A, Kishi C, Waguri S, et al. Autophagydeficient mice develop multiple liver tumors. Genes Dev. 2011;25:795-800.

50. Wang S, Jiang W, Chen X, Zhang C, Li H, Hou W, et al. Alpha-fetoprotein acts as a novel signal molecule and mediates transcription of Fn14 in human hepatocellular carcinoma. J Hepatol. 2012;57:322-9.

\section{ACKNOWLEDGEMENTS}

We thank Associate Professor Tingting Li (Department of Biomedical Informatics, Peking University Health Science Center) for her suggestions about bioinformatic analysis including KEGG pathway enrichment analysis and GO functional enrichment analysis.

\section{AUTHOR CONTRIBUTIONS}

$\mathrm{HH}$ and $\mathrm{QH}$ contributed equally to this work. JW and $\mathrm{HG}$ designed the research; $\mathrm{HH}$, $\mathrm{QH}, J W, H G, H Z, M L, S S, T Z, X Y, Z L$, and $Q X$, performed the research; all authors analyzed the data; $\mathrm{HH}, \mathrm{JW}$, and $\mathrm{QH}$ wrote the paper; JW, $\mathrm{HG}$, and $\mathrm{FL}$ revised the paper.

\section{FUNDING}

This work was supported by grants from the National Natural Science Foundation of China (No. 81672741 and 81974309).

\section{ETHICS STATEMENT}

The in vivo study in nude mice was approved by the Ethics Committee of Peking University Health Science Center. Study on the tumor samples of cervical cancer patients was approved by the Ethics Committee of Third Hospital of Peking
University. Written informed consent was obtained from each patient. All procedures were in accordance with the ethical standards.

\section{COMPETING INTERESTS}

The authors declare no competing interests.

\section{ADDITIONAL INFORMATION}

Supplementary information The online version contains supplementary material available at https://doi.org/10.1038/s41419-021-04474-1.

Correspondence and requests for materials should be addressed to Hongyan Guo or Jie Wang.

Reprints and permission information is available at http://www.nature.com/ reprints

Publisher's note Springer Nature remains neutral with regard to jurisdictional claims in published maps and institutional affiliations. (c) Open Access This article is licensed under a Creative Commons cc) Attribution 4.0 International License, which permits use, sharing, adaptation, distribution and reproduction in any medium or format, as long as you give appropriate credit to the original author(s) and the source, provide a link to the Creative Commons license, and indicate if changes were made. The images or other third party material in this article are included in the article's Creative Commons license, unless indicated otherwise in a credit line to the material. If material is not included in the article's Creative Commons license and your intended use is not permitted by statutory regulation or exceeds the permitted use, you will need to obtain permission directly from the copyright holder. To view a copy of this license, visit http://creativecommons. org/licenses/by/4.0/.

(c) The Author(s) 2021 